

\section{ENGINEERING CHANGE NOTICE}

Page 2 of $Z$

1. ECN (use no. from pg. 1)

16. Design Verification 17 . Cost Impact

Required

ENGINEERING

Yes

ONo
S $N / A$

O $N / A$

\section{CONSTRUCTION}

Additional $\bigcirc \leqslant \underline{N} / \mathrm{A}$

Savings $O \leqslant N / A$
644049

18. Schedule Impact (days)

Improvement

$\mathrm{N} / \mathrm{A}$

Delay

$\mathrm{N} / \mathrm{A}$

19. Change Impact Review: Indicate the related documents (other than the engineering documents identified on Side 1) that will be affected by the chiange described in Block 13. Enter the affected document number in Block 20.

SDDIDD

Functional Design Criteria

Operating Specification

Criticality Specification

Conceptual Design Report

Equipment Spec.

Const. Spec.

Procurement Spec.

Vendor Information

OM Manual

FSAR/SAR

Safety Equipment List

Radiation Work Permit

Environmental Impact Statement

Environmental Report

Environmental Permit $\square$

$\square$

$\square$

$\square$

$\square$

$\square$

$\square$

$\square$

$\square$

$\square$

$\square$

$\square$

$\square$

$\square$

$\square$

$\square$

$\square$
Seismic/Stress Analysis

Stress/Design Report

Interface Control Drawing

Calibration Procedure

Installation Procedure

Maintenance Procedure

Engineering Procedure

Operating Instruction

Operating Procedure

Operational Safety Requirement

IEFD Drawing

Cell Arrangement Drawing

Essential Material Specification

Fac. Proc. Samp. Schedule

Inspection Plan

Inventory Adjustment Request $\square$ Tank Calibration Manual

$\square \quad$ Health Physics Procedure

$\square \quad$ Spares Multiple Unit Listing

$\square$ Test Procedures/Specification

$\square$ Component Index

$\square \quad$ ASME Coded Item

$\square \quad$ Human Factor Consideration

$\square \quad$ Computer Software

$\square \quad$ Electric Circuit Schedule

$\square \quad$ ICRS Procedure

$\square \quad$ Process Control Manual/Plan

$\square \quad$ Process Flow Chart

$\square \quad$ Purchase Requisition

Tickler File

$\square \quad$ None

None

20. Other Affected Documents: (NOTE: Documents listed below will not be revised by this ECN.) Signatures below indicate that the signing organization has been notified of other affected documents listed below.

Document Number/Revision

Document Number/Revision

Document Number/Revision

None

\section{Approvals}

Signature

Design Authority B.D, Groth 894 toth

Cog. Eng.

Cog. Mgr.

QA

Safety

Environ.

Other
Date $10 / 7 / 9)$

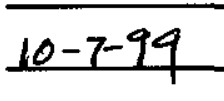


HNF-4460, Rev. 1

\section{Design Package Test Weights For Fuel Retrieval System (OCRWM)}

\section{David Tedeschi}

for Duke Engineering and Services Hanford, Richland, WA 99352

U.S. Department of Energy Contract DE-AC06-96RL13200

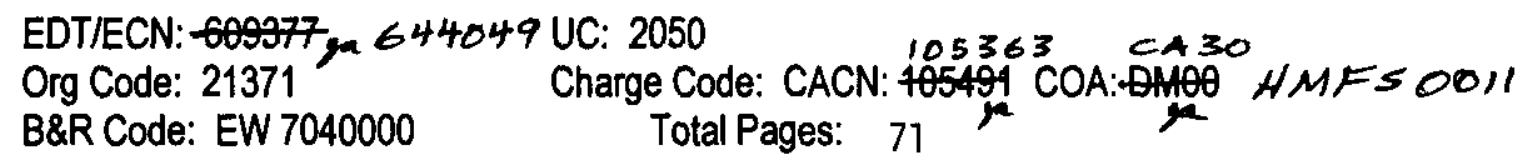

Key Words: Spent Nuclear Fuel, Fuel Retrieval System, Weights, Test

Abstract: This is a design package that documents the development of test weights used in the Spent Nuclear Fuels subproject Fuel Retrieval System.

TRADEMARK DISCLAIMER. Reference herein to any specific commercial product, process, or service by trade name, trademark, manufacturer, or otherwise, does not necessarily constitute or imply its endorsement, recommendation, or favoring by the United States Government or any agency thereof or its contractors or subcontractors.

Printed in the United States of America. To obtain copies of this document, contact: Document Control Services, P.O. Box 950, Mailstop H6-08, Richland WA 99352, Phone (509) 372-2420; Fax (509) 376-4989.

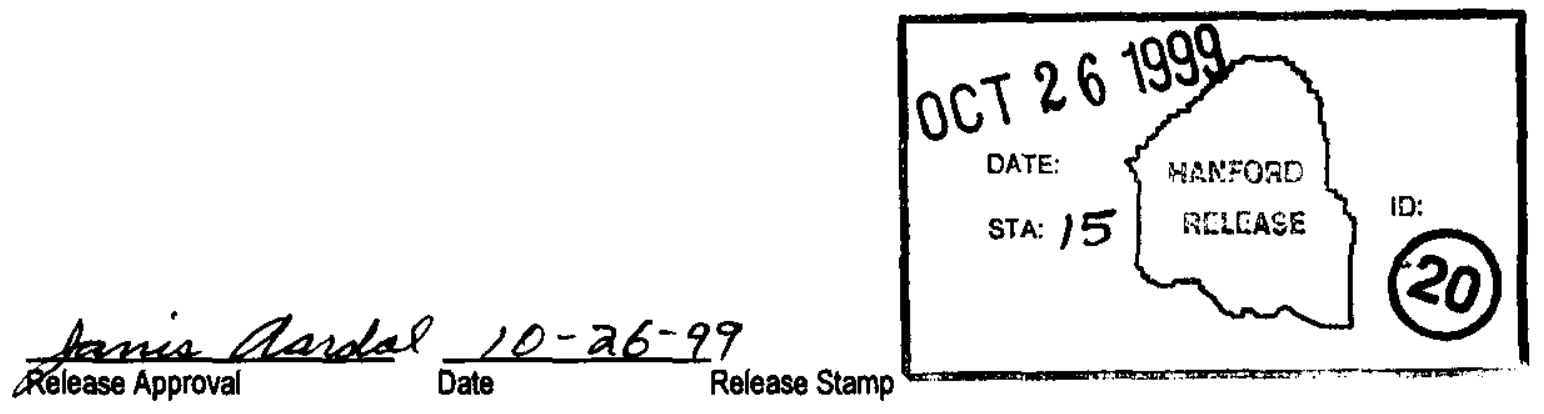

\section{Approved for Public Release}




\section{RECORD OF REVISION}

(2) Title

Design Package Test Weights for Fuel Retrieval system

Change Control Record

(3) Revision

1 RS
(4) Description of Change - Replace, Add, and Delete Pages

\section{(7)}

Added in Appendix B information

Added in information to verification section on

the testing/calibration Usuc ECN 644049
Authorized for Release

\begin{tabular}{|l|l|}
\hline (5) Cog. Engr. & (6) Cpg. Mgr. Date \\
\hline
\end{tabular} $6 / 7$ ha

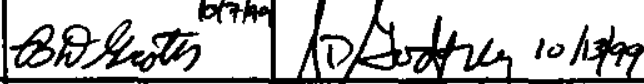

BD Groth so Godfrey 


\title{
Design Package
}

\section{Test Weights for Fuel Retrieval System (OCRWM) \\ HNF-4460, Rev 1}

August 25, 1999

By H. L. Roach

Of

ElvorDaniel Northwest

1200 Jadwin

Richland, WA 99352

\author{
For Bruce Groth \\ Representing \\ Duke Enginéring \& Services Company Hanford \\ P.O. Box 350 \\ Richland, WA 99352
}




\subsection{Introduction}

The K Basins Spent Nuclear Fuel (SNF) project consists of the safe retrieval, preparation, and repackaging of the spent fuel stored at the $\mathrm{K}$ East $(\mathrm{KE})$ and $\mathrm{K}$ West (KW) Basins for interim safe storage in the Canister Storage Building (CSB).

Multi-Canister Overpack (MCO) scrap baskets and fuel baskets will be loaded and weighed under water. The equipment used to weigh the loaded fuel baskets requires daily calibration checks, using test weights traceable to National Institute of Standards Testing (NIST) standards. The test weights have been designated as OCRWM related in accordance with HNF-SD-SNF-RPT-007 (McCormack).

\subsection{Scope}

\section{$2.1 \quad$ Objectives}

The scope of this design package is to document the design and fabrication of two NIST traceable test weights to be used for the daily calibration of the load measuring equipment on the monorail mounted MCO Stiffback grapple.

\subsection{Products Delivered}

This package will deliver the following:

\section{Documents}

a) This document, which includes product requirements, design analysis, graphical depictions, and calibrations reports.

b) Hanford formatted detail and assembly drawings.

\section{Hardware}

a) NIST traceable test weight. Dry weight will be $1560.4 \mathrm{~kg}(\sim 3437 \mathrm{lbs}$.). Underwater weight will be $1362 \mathrm{~kg}(\sim 3000 \mathrm{lbs}$.). Weight will be fabricated to $+/-0.5 \%$ of the underwater weight.

b) NIST traceable test weight. Dry weight will be $654 \mathrm{~kg}(\sim 1440 \mathrm{lbs}$.$) .$ Underwater weight will be $570 \mathrm{~kg}(\sim 1256 \mathrm{lbs}$.). Weight will be fabricated to $+/-0.5 \%$ of the underwater weight.

c) Lifting beam to place the test weights in their storage locations at $\mathrm{K}$ Basins using a standard 1 ton chain fall or hoist with standard hook. 


\subsection{Products Requirements and Constraints}

The general design requirements/criteria for the Spent Nuclear Fuel Project Fuel Retrieval subproject is in HNF-S-0461, Specification for Design of the SNF Project Fuel Retrieval Subproject. The following are additional requirements:

\subsection{Dimensional/Physical Constraints}

a) Diameter of test weights will be $22.25+/-.010$ inches.

b) Maximum height shall be less than 33.75 inches (See figure 1).

c) No part of the test weights may weigh more than $771.8 \mathrm{~kg}$ (1700 lbs.). (HNF-2229, Appendix J).

d) Weights must fit storage locations along monorail no. 27 on the Process Support Table, BNFL drawing DW-327, rev. 6.

\subsection{Material Requirements}

K Basins Design Guidelines (Roe 1995) shall be used as the source document for establishing material design requirements. Material requirements that pertain to the test weight construction are as follows:

- Carbon steel is an acceptable material for $\mathrm{K}$ Basin equipment. Carbon steel, if used, shall be coated to prevent corrosion and/or ease decontamination. Coating shall consist of a general Amercoat ${ }^{\mathrm{TM}}$ epoxy system consisting of Amercoat ${ }^{\mathrm{TM}}$ Number 64 primer and Amercoat ${ }^{\mathrm{TM}}$ Number 66 seal gloss topcoat. ${ }^{1}$

- 300 Series Stainless Steel (SST) is an acceptable material. 300 Series SST shall be used for lifting beams and/or strongbacks.

- If lead is used it will be encapsulated to prevent contamination of the lead.

\subsection{Environmental Constraints}

a) Test weight material must be able to withstand the effects of high radiation field of $40 \mathrm{rem} / \mathrm{hr}$.

\footnotetext{
${ }^{1}$ Amercoat ${ }^{\mathrm{TM}}$ is a registered trademark of Ameron protective Coatings Group, Brea Calif.
} 


\section{Fuel Retrieval System Small Tools Design Package}

Test Weights

HNF-4460, Rev 1

August 25, 1999

Page 4

b) Test weight design shall accommodate $46-53^{\circ} \mathrm{F}$ basin water temperatures.

c) Test weight materials shall be compatible with the deionized/demineralized water.

\subsection{Operational Requirements}

a) Test weights will be designed for low corrosion during continuous underwater storage at K-Basin.

b) The weight accuracy tolerance will not be exceeded from corrosion of the test weights over the design life of the test weights.

c) Weights will be lowered into the K-Basin, moved and placed in their storage location using a monorail hoist system with a standard 1 ton chain fall or hoist with a standard hook.

d) Weights must interface with the fuel basket grapple, H-1-82864, rev. 0 , sheets 1 and 2, and BNFL drawing DW-209. Fuel basket grapple will be used to lift test weight during load cell calibration.

e) Specified test weight, $1362 \mathrm{~kg}(3000 \mathrm{lb}$.) and $570 \mathrm{~kg}(1256 \mathrm{lb}$.) shall be as measured under water. Fabrication shall be to $+/-0.5 \%$ of the specified weight. Design shall take into account the buoyant effect of displaced water.

f) Design life shall be 10 years. Frequency of use will be daily.

\subsection{Equipment Marking Requirements}

a) Below the hook lifting devices used with the test weights shall be marked as follows:

- The number(s) of the $\mathrm{H}-1$ drawing used for the equipment design and construction.

- Weight (If over 100 lbs.)

- Rated load. 


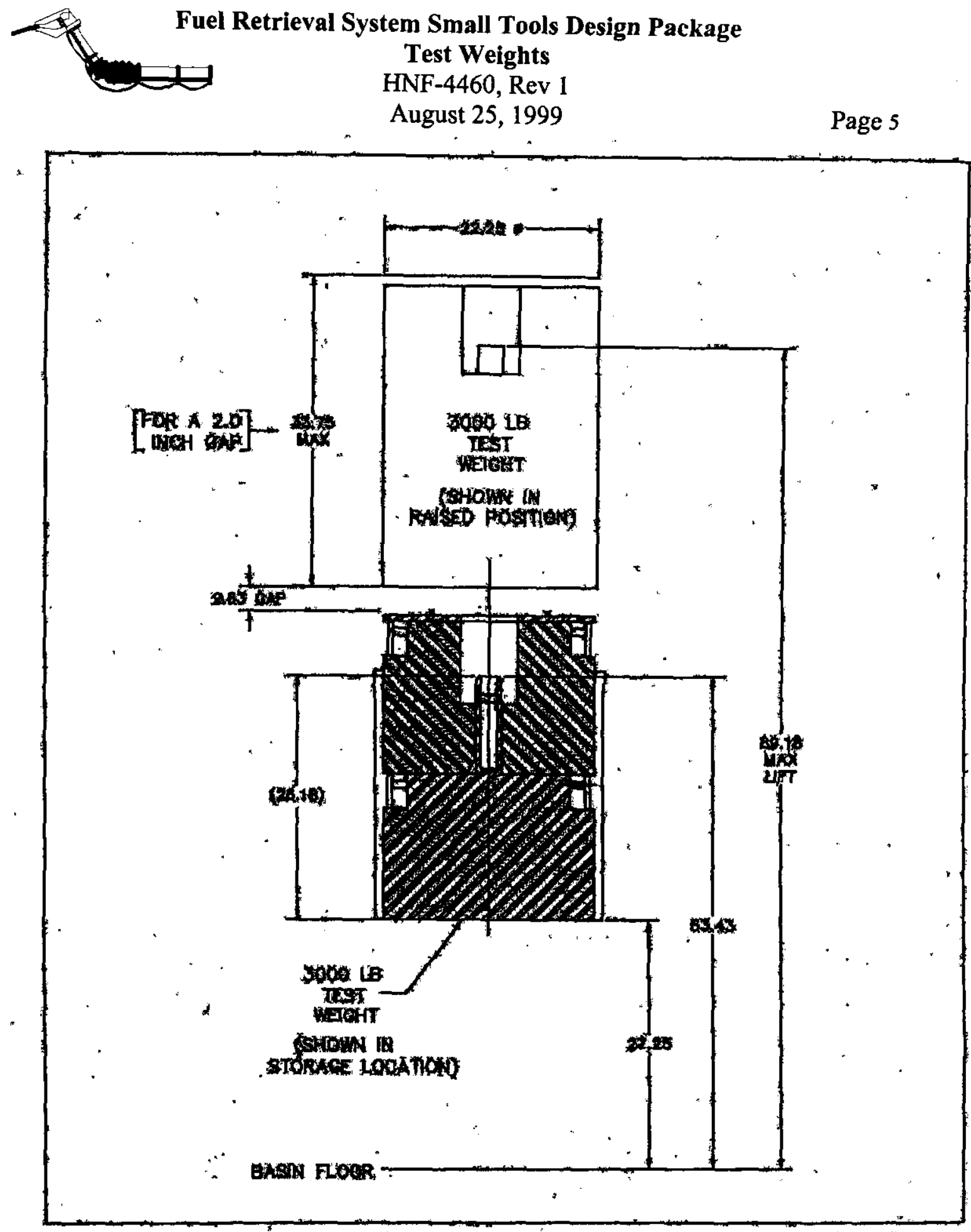

FIGURE 1 - MAX HEIGHT OF WEIGHT 


\section{Fuel Retrieval System Small Tools Design Package}

Test Weights

HNF-4460, Rev 1

August 25, 1999

Page 6

\subsection{Calibration Requirements}

a) Weights shall be certified for underwater weight using a source traceable to NIST standards.

b) Test weights shall be certified to $+/-0.5 \%$ of its rated underwater weight.

\subsection{Design Stress Criteria}

a) Test weights shall be designed with a design factor of 3.0 based on the yield stress of the material.

b) Below the hook lifting devices will be designed in accordance ANSI B30.20, Below the Hook Lifting Devices.

\subsection{Welding Requirements}

All welding shall be in accordance with ANSI/AWS D1.1, Structural Welding code.

\subsection{Maintenance Requirements}

Equipment shall be designed for no maintenance.

\subsection{Applicable Laws, Regulations, and Standards}

The applicable Fluor Daniel Hanford Engineering procedures for documenting design, review, and approval of engineering documents shall be used.

\subsection{Development of Product}

\subsection{Management of Task}

\subsubsection{Engineering}

Engineering design, design analysis, and preparation of Hanford formatted H-1 drawings will be done by Fluor Daniel Northwest (FDNW). Documents will be reviewed and approved by Duke Engineering \& Services Hanford. 


\subsubsection{Fabrication / Calibration}

FDNW will be responsible for fabrication of the test weights and supporting equipment. In addition FDNW will be responsible for initial certification of the test weights.

\subsection{Development}

\subsubsection{Conceptual Design}

The conceptual design for the two test weight assemblies are shown in Appendix A of the calculation and is listed as Figures $\mathrm{A} 1$, and $\mathrm{A} 2$.

The 1362-kg ( $3000 \mathrm{lb}$. measured underwater) test weight assembly is showed in Appendix A, Figure A1. It is fabricated entirely of 304 SST. It is necessary to fabricate this weight in three pieces to meet the requirement that no part of the test weight can weigh more than $1700 \mathrm{lbs}$. (in air). Size, shape, dry and underwater weight, and surface areas are shown. It is anticipated that the test weight can be fabricated from thick plate or solid billet (if available).

The 570-kg (1256 lb. measured underwater) test weight assembly is showed in Appendix A, Figure A2. It is also fabricated entirely of 304 SST. Size and shape, dry and underwater weight, and surface areas are shown. Method of fabrication will be the same as for the other test weight.

The Lifting beam is used to lower the test weight sections into the $\mathrm{K}$-Basin using a standard 1 ton crane or chain hoist and is shown in Appendix A, Figure A3. The largest weight that will be lifted using this lifting beam is $1700 \mathrm{lbs}$. It was designed for a $1800 \mathrm{lb}$ maximum load to give it a capacity slightly larger than what is required. It is constructed entirely of 304 SST as required by the K Basin design guidelines (Roe 1995).

\subsubsection{Final Design}

The final design was documented on a drawing titled "FRS In-Pool Equipment Test Weights" and its number is H-1-83994. It was fabricated in the DYN Corp (Hanford site fabrication shops) under work package number $2 \mathrm{H} 9903763$. It was tested and certified at the Numatec operated 305 test facility. 


\section{Fuel Retrieval System Small Tools Design Package}

Test Weights

HNF-4460, Rev 1

August 25, 1999

Page 8

Of significant note is that the third piece of the $3000 \mathrm{lb}$. weight was removed from the design in order to improve ease of installation. This resulted in a calibrated weight of $2954 \mathrm{lbs}$. in water. This is acceptable as long as it is certified to within $\pm 0.5 \%$ of actual weight.

\subsection{Verification of Product Design}

The test weights were verified through testing and calculations. Calculations were used to verify those items, which could not be reasonably tested. The calculations focused on the environmental issues such as corrosion. The testing verified that the design weight was met in and out of water. The test verification produced a documented certified calibration for each piece if the weight.

\subsubsection{Design Calculations}

Design calculations are included in Appendix A. The following calculations were made:

- Evaluation of corrosion on accuracy of test weights

- Evaluation of basin water temperature on test weight accuracy

- Evaluation of material and weld stresses

Calculations were made to evaluate the impact of corrosion of the test weight during the 10-year design life of the test weights on the accuracy of the test weights. The calculations showed that the corrosion of the test weights over a 10-year design life resulted in a $0.08 \%$ (max) decrease in the weight of the test weight. This will not impact the required accuracy of the test weights (see Appendix $A, 2.6)$. If the test weights are re-calibrated during the 10 year period corrosion will be even more insignificant since recalibration will account for material loss since the last calibration.

Likewise the variation of the basin water temperature proved to have a very small impact on the measured weight of the test weights. Temperature variations will result in a $0.008 \%$ variation in measured weight. The calculation made is in Appendix A, 2.6, and shows that basin water temperature can be ignored. 


\subsubsection{Testing/Calibration}

Testing/calibration was performed by Energy Northwest Standards Laboratory. A Fluor Daniel Northwest (FDNW) Design engineer provided a statement of work which provide the testing steps that they needed to perform. The FDNW engineer observed all the steps in the performance of the calibration. The statement of work along with the data sheet is in Appendix B.

The weights were weighed three times with a calibrated dynamometer in dry air and in the water and an average weight was calculated. The calibration data for the dynamometer is in Appendix B. The average weight for each of the weights did not deviate at all. For instance, if $1436 \mathrm{lbf}$ was measured, then the next two measurements showed the same value. All the measurements showed the weights were in tolerance too. See Table 1 for test data. The actual data sheets are listed in Appendix B. Final test data is as follows:

Table 1. Test Data

\begin{tabular}{|c|c|c|}
\hline [10m & $\begin{array}{l}* \text { W cight in air } \\
\text { (Il) })+(1.5 \% / 1\end{array}$ & $\begin{array}{l}* \text { W eight in " alter } \\
\text { (ll)f) } \pm(1.5 \% / \%\end{array}$ \\
\hline $\mathrm{H}-1-83994-02$ & 1436 & 1256 \\
\hline H-1-83994-03 & 1692 & \\
\hline H-1-83994-04 & 1686 & \\
\hline $\begin{array}{l}\text { Combined weight } \\
\text { of item } 03 \text { and } 04\end{array}$ & 3378 & \\
\hline
\end{tabular}

* Weights shown are the average weight.

\subsection{Conclusion}

The test weights were designed as specified by the requirements and are documented on the drawing numbered H-1-83994. The weights have been calibrated and verified to meet the designed weight within tolerance. The Energy Northwest calibration recall numbers are 999-86-02-103, 999-8602-104, and 999-86-02-105. 


\subsection{References}

AWS, 1996, Structural Welding Code, AWS D1.1, D1.2, D1.3, American Welding Society, Miami, Florida

ANSI, 1985, Below the Hook Lifting Devices, ANSI B30.20-85, American National Standards Institute, New York, NY

Drawing, $K$-Basin SNF Storage Basket Mark 1A, H-2-828060, Rev 1, Duke Engineering \& Services Hanford, Richland Washington

Drawing, Fuel Basket Grapple End Effector Assembly, H-1-82864, Rev 0, MCE Engineering, Richland Washington

Drawing, FRS In-Pool Equipment Process Table Supports Arrangement, DW-327, Rev 6, British Nuclear Fuels Ltd., Richland, WA

Duncan, D.R., 1998, Submerged Weight of Scrap Baskets Issue Closure Package, HNF-3058, Rev. 0, Duke Engineering \& Services Hanford, Richland Washington

Henderson, J.M., 1998, Fuel Retrieval Sub Project Project Analysis Report for Safety Class in Pool Equipment, HNF-2229, Rev 0, British Nuclear Fuels Ltd., Richland, WA

Groth, B.D., 1998, Specification for Design of the SNF Project Fuel Retrieval Subproject, HNF-S-0461, Rev. 1, Duke Engineering \& Services Hanford, Richland, Washington

Roe, N.R., 1995, K Basins Design Guidelines, WHC-SD-SNF-DGS-001, rev. 0, Westinghouse Hanford Company, Richland, Washington

McCormack, R.L., 1999, HNF-SD-SNF-RPT-007, Application of the Office of Civilian Radioactive Waste Management QA Requirements to the Hanford Spent Nuclear Fuel Project, Duke Engineering and Services Hanford, Richland, Washington 
Fuel Retrieval System Small Tools Design Package

Test Weights

HNF-4460, Rev 1

August 25, 1999

Page $A-0$

Appendix A

Design Calculations 
HNF-4460 Rev 1

Page A-1

The following analyses are verifications for the design of the test weights for the Fuel Retrieval System. 
FLUOR DANIEL NORTHWEST, INC.

HNF-4460, Rev 1

CALCULATIONS AND SKETCHES SHEET

\begin{tabular}{llll} 
DEPARTMENT: & $\begin{array}{c}\text { Mechanisms } \\
\text { Engineering }\end{array}$ & ORIGINATED BY: H.L.Roach & DATE: $5 / 20 / 99$ \\
\hline ENG COMM NO: & & CHECKED BY: & REVISED BY:
\end{tabular}

SUBJECT: Test Weights for K-Basin

Client: Duke Engineering \& Services Company Hanford

Task Order Number: 651003312 F04

Design Calculations for K-Basin Test Welghts

ORIGINATED BY: H.L. Roach

CHECKED BY: R.G. Hollenbeck 
FLUOR DANIEL NORTHWEST, INC.

HNF-4460, Rev 1

CALCULATIONS AND SKETCHES SHEET

\begin{tabular}{|c|c|c|c|}
\hline DEPARTMENT: & $\begin{array}{l}\text { Mechanisms } \\
\text { Engineering }\end{array}$ & ORIGINATED BY: H.L.Roagh heh & $5 / 20 / 99$ \\
\hline ENG COMM NO: & & SPll ll e $k^{\text {DATE: }}$ & $5 / 20 / 90$ \\
\hline AREA: & $100 \mathrm{~K}$ & REVISED BY: & \\
\hline
\end{tabular}

\subsection{Purpose}

The purpose of these calculations is to calculate the following for the Fuel Retrieval System test weights.

- Conceptual design of 304 Stainless Steel (SST) $3000 \mathrm{lb}$. test weight. Calculate dry weight, underwater weight, and surface areas.

- Conceptual design of 304 SST $1256 \mathrm{lb}$. $(570 \mathrm{~kg})$. test weight. Calculate dry weight, underwater weight, and surface areas.

- Conceptual design of lifting beam for test weights.

- Evaluate effect of corrosion on accuracy of test weights

- Evaluate effect of basin water temperature on accuracy of test weights.

- Evaluate material and weld stresses for conceptual Design.

\subsection{Conceptual Design Physical Properties}

2.1 Conceptual Design and Physical Properties of 3000 LB Test Weight

Figure A1 shows the proposed conceptual design for a 3000-LB test Weight. The following summarizes the physical properties of this design:

- All stainless steel construction

- Three part design

- No part weighs over 1700 LBS.

- Overall height is $32.62^{n}$

- Diameter is $22.25^{\prime \prime}$

- Combined underwater weight is $\sim 3000 \mathrm{lbs}$.

- Total exposed surface areas is $\sim 4912.2$ in $^{2}$.

- Volume is $\sim 12042 \mathrm{in}^{3}$ 
FLUOR DANIEL NORTHWEST, INC.

HNF-4460, Rev 1

CALCULATIONS AND SKETCHES SHEET

\begin{tabular}{|c|c|c|c|c|}
\hline DEPARTMENT: & $\begin{array}{l}\text { Mechanisms } \\
\text { Engineering }\end{array}$ & ORIGINATED BY: & DATE: & $5 / 26 / 99$ \\
\hline ENG COMM NO: & & & DATE: & \\
\hline
\end{tabular}

SUBJECT: Test Wolghts for K-Basin

THARD PART

DRY WEGHT: 80.70 LOS

UNDERWATER WIGAT: 49.52 LAS

SURPACE AREA 770.00 NA2

SECOND RART

DRY WJGH7: 1090.03 LAS

UNDERWATR WJGHT: 1473.25 LAS

SURFACE AREA $2130.60 \mathrm{NN}^{\wedge 2}$

BAFE PART

DSY 1890.01 LAS

UAOJRWATER WEGHT: 1475.24 LAS

SUFFACE AREA N $2002.49 \mathrm{Wh}$
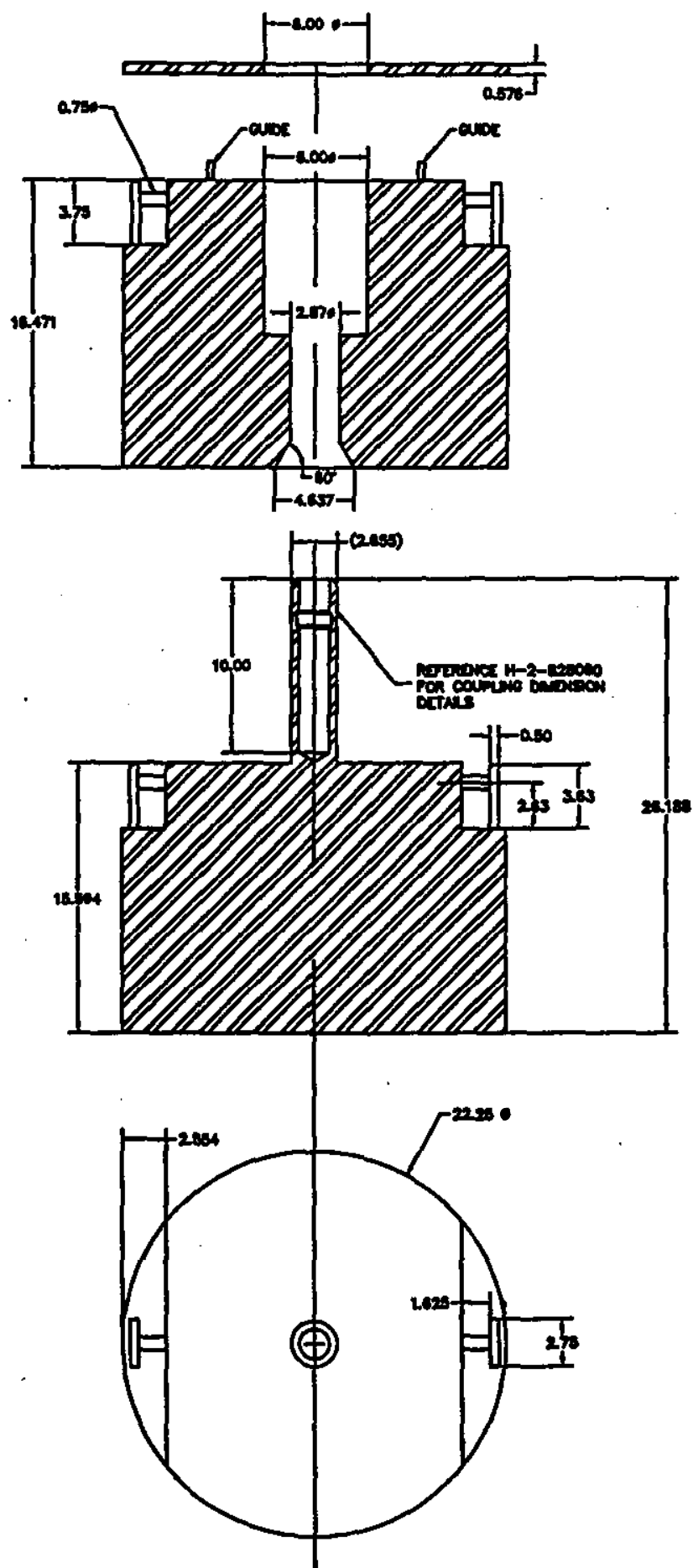

Page A4

EIGURE A1 - 3000 LB SOLID 304 SST

304 STANrixs $5 T 0 x$

All WinNo Is ANS/Aws Dt

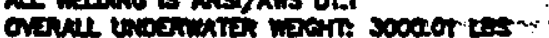

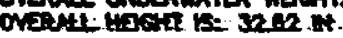


FLUOR DANIEL NORTHWEST, INC.

CALCULATIONS AND SKETCHES SHEET

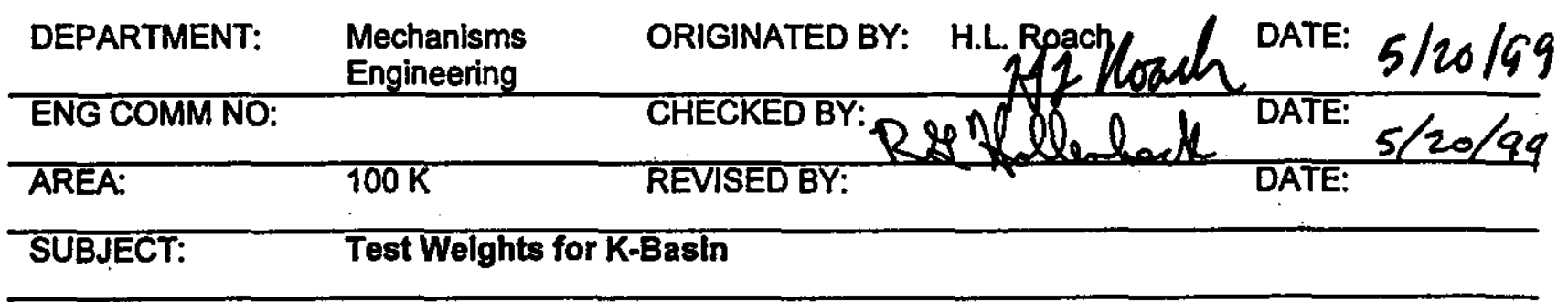

Numbers Based on Solid Model Development using AutoCAD R14

\section{Test Wejght Work Sheet - Solid Steel}

\begin{tabular}{|c|c|c|}
\hline owntsity & alcings & 6in \\
\hline $\begin{array}{l}304 \text { SST } \\
\text { Water @ } 50 \mathrm{~F}\end{array}$ & $\begin{array}{l}7.900 \\
0.9997\end{array}$ & $\begin{array}{l}0.285 \\
0.036\end{array}$ \\
\hline
\end{tabular}

- Source CRC Handbook of Chemistry and Physics, 77th Edition, CRC Press, Boca Raton, Filorita

LLfting Attachment Volume and Weight Calcs

Consists of $0.75^{\prime \prime}$ OD Rod and $0.50^{\prime \prime}$ Plate (304 SST)

Rod

Dia (in) Length (in) Vol (in^3) Lb

$\begin{array}{llll}0.750 & 1.625 & 0.718 & 0.205\end{array}$

Plate

Length (in) Width (in) Thick (in) Vol (in^3)

(DRY)

$\begin{array}{lllll}2.750 & 3.630 & 0.500 & 4.991 & 1.422\end{array}$

Total Volume: $5.709 \quad \ln ^{\wedge 3}$

Total Weight: 1.627 lbs

Figure A1 - Development of Base of 3000 LB Welght

Helght is $11.875^{\prime \prime}$

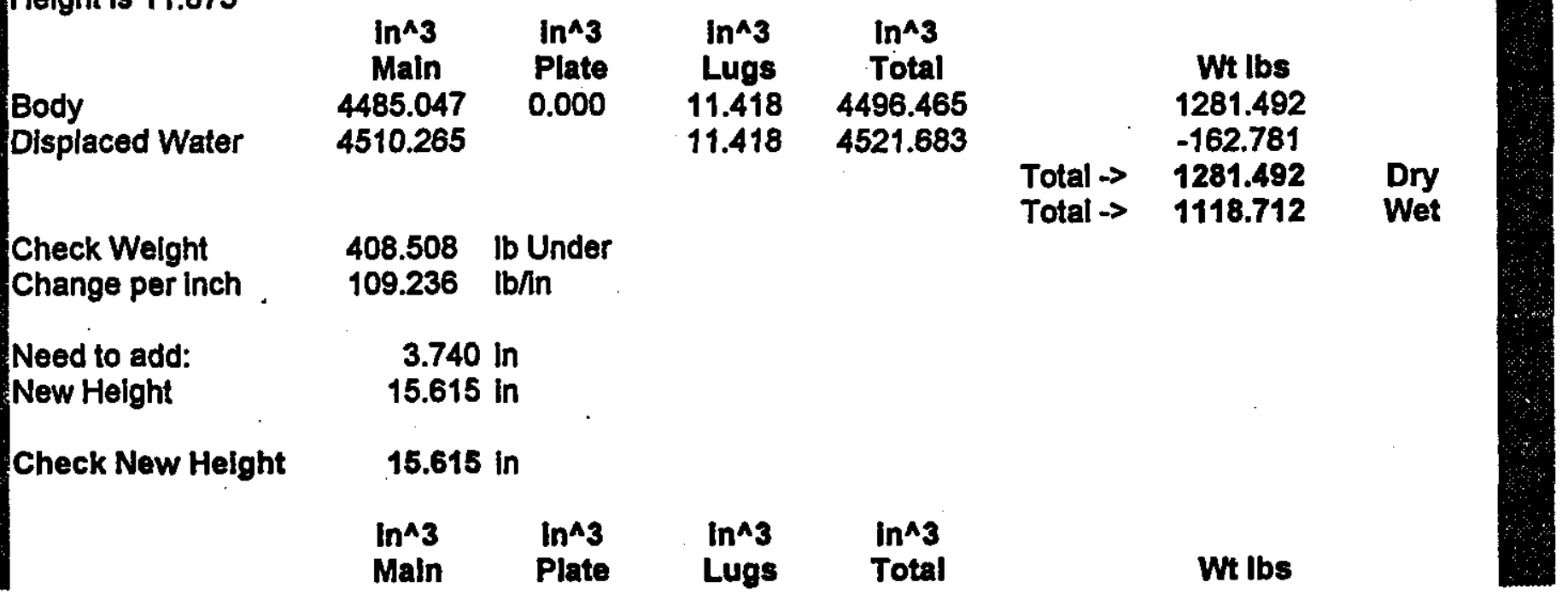


FLUOR DANIEL NORTHWEST, INC.

HNF-4460, Rev 1

CALCULATIONS AND SKETCHES SHEET

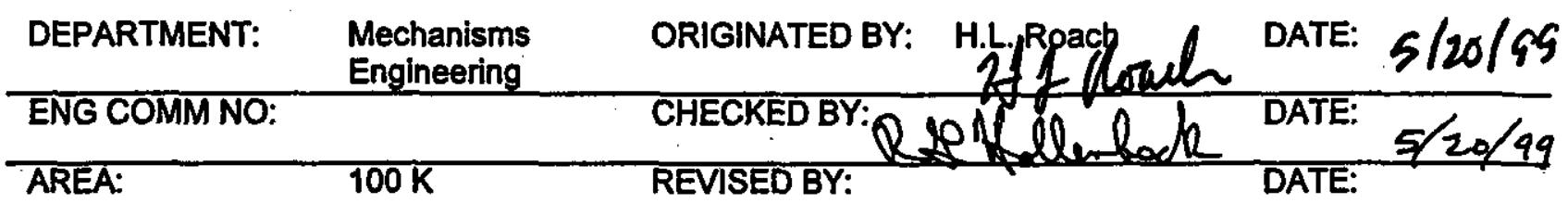

SUBJECT: Test Weights for K-Basin

Body

Displaced Water

5910.561

5935.779

OKI Dry Weight is less than 1700 LBS
11.418

11.418
5921.979
5947.197

5947.197
1687.764

$-214.099$

Total $\rightarrow \quad 1687.764$

Total $\rightarrow \quad 1473.665$
Dry

Wet

\section{Flgure A1 - Development of 2nd Part of 3000 LB Welght}

Height is 18"

Body

Displaced Water

Target Weight

Check Weight

Calculate 1" slice

Need to add:

New Height

Check New Height

(T)

Steel Shell with cutout

Displaced Water
18 in

\begin{tabular}{|c|c|c|c|}
\hline $\begin{array}{c}\text { In^3 } \\
\text { Maln } \\
6461.881 \\
6461.881\end{array}$ & $\begin{array}{l}\ln ^{1} 3 \\
\text { Plate } \\
0.000\end{array}$ & $\begin{array}{r}\text { in^3 } \\
\text { Lugs } \\
11.418 \\
11.418\end{array}$ & $\begin{array}{c}\text { in^3 }^{\wedge} \\
\text { Total } \\
6473.299 \\
6473.299\end{array}$ \\
\hline
\end{tabular}

$\begin{array}{cc}1690.000 & \text { lb (dry) } \\ 154.890 & \text { lb Over } \\ 102.756 & \text { lb/in }\end{array}$

-1.507 in

16.493 in

16.493 in

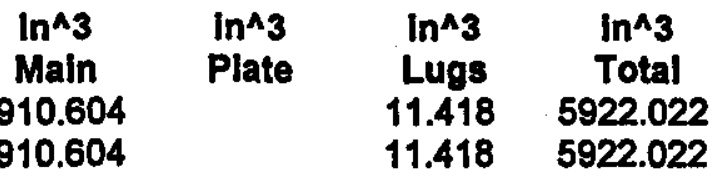

Wt Ibs

1844.890

$-233.039$

Total $\rightarrow \quad 1844.890$

Total $\rightarrow \quad 1611.851$
Dry

Wet

\section{OKI Dry Weight is less than 1700 LBS}

$\begin{array}{lcc} & \text { Wt lbs } & \\ & 1687.776 & \\ & -213.193 & \\ & \\ \text { Total } \rightarrow> & 1687.776 & \text { Dry } \\ \text { Total } \rightarrow & 1474.583 & \text { Wet }\end{array}$

\section{Flgure A1 - Development of 3rd Part of 3000 LB Weight}

Check against $3000 \mathrm{tb}$ target

Total First Two Parts

2948.248 lbs (Wet)

Target Weight

Need to Add

3000.000 lbs (Wet)

Need Slice

$\mathbf{5 1 . 7 5 2}$ lbs (Wet)

0.576 Inches - Estimate

Height is 0.576 in

In ${ }^{\wedge} \quad \ln ^{\wedge} 3 \quad \ln ^{\wedge} 3 \quad \ln ^{\wedge} 3$ 
FLUOR DANIEL NORTHWEST, INC.

HNF-4460, Rev 1

CALCULATIONS AND SKETCHES SHEET

\begin{tabular}{lll} 
DEPARTMENT: & $\begin{array}{l}\text { Mechanisms } \\
\text { Engineering }\end{array}$ & ORIGINATED BY: H.L.Roach \\
\hline ENG COMM NO: & & CHECKED BV \\
AREA: & $100 \mathrm{~K}$ & REVISED BY:
\end{tabular}

SUBJECT: Test Weights for K-Basin

\begin{tabular}{|c|c|c|c|c|c|c|c|}
\hline $\begin{array}{l}\text { Body } \\
\text { Displaced Water }\end{array}$ & $\begin{array}{c}\text { Main } \\
207.675 \\
207.675\end{array}$ & $\begin{array}{l}\text { Plate } \\
0.000\end{array}$ & $\begin{array}{l}\text { Lugs } \\
0.000 \\
0.000\end{array}$ & $\begin{array}{c}\text { Total } \\
207.675 \\
207.675\end{array}$ & $\begin{array}{l}\text { Total -> } \\
\text { Total } \rightarrow>\end{array}$ & $\begin{array}{c}\text { Wt lbs } \\
59.187 \\
-7.476 \\
59.187 \\
51.711\end{array}$ & $\begin{array}{l}\text { Dry } \\
\text { Wet }\end{array}$ \\
\hline $\begin{array}{l}\text { Tanget Weight } \\
\text { Check Weight } \\
\text { Calculate Whin }\end{array}$ & $\begin{array}{l}51.752 \\
-0.041 \\
89.776\end{array}$ & $\begin{array}{c}\text { Ib (Wet) } \\
\text { lb } \\
\mathrm{lb} / \mathrm{hn}\end{array}$ & & & & & \\
\hline $\begin{array}{l}\text { Add additional height } \\
\text { New Helght }\end{array}$ & $\begin{array}{l}0.00 \\
0.57\end{array}$ & & & & & & $\cdot$ \\
\hline
\end{tabular}

Page A7 
FLUOR DANIEL NORTHWEST, INC.

HNF-4460, Ret 1

CALCULATIONS AND SKETCHES SHEET

\begin{tabular}{|c|c|c|c|c|}
\hline DEPARTMENT: & $\begin{array}{l}\text { Mechanisms } \\
\text { Engineering }\end{array}$ & ORIGINATED BY: H.L & DATE: & 5120199 \\
\hline ENG COMMNO: & & CHECKED BY: & DATE: & $5 / 2$ \\
\hline AREA: & $100 \mathrm{~K}$ & REVISED BY: & DATE: & \\
\hline
\end{tabular}

2.2 Conceptual Design and Physical Properties of $1256 \mathrm{lb} .(570 \mathrm{~kg})$ Test Weight

Figure A2 shows the proposed conceptual design for a $1256 \mathrm{lb}$. (570 $\mathrm{kg})$ test Weight. The following summarizes the physical properties of this design:

- All stainless steel construction

- One piece design

- Test weight weighs 1439.8 LBS in air.

- Overall height is $26.19^{\prime \prime}$

- Diameter is $22.25^{n}$

- Combined underwater weight is $\sim 570 \mathrm{~kg}$ (1256 lbs.).

- Total exposed surface areas is 1861.64 in $^{2}$

- Volume is $\sim 5044.43$ in $^{3}$ 
FLUOR DANIEL NORTHWEST, INC.

HNF-4460, Rov 1

CALCULATIONS AND SKETCHES SHEET

\begin{tabular}{|c|c|c|c|c|}
\hline DEPARTMENT: & $\begin{array}{l}\text { Mechanisms } \\
\text { Engineering }\end{array}$ & ORIGINATED BY: & DATE: & $5 / 20 / 99$ \\
\hline ENG COMMNO: & & KED & DATE: & $5 / 20$ \\
\hline
\end{tabular}

SUBJECT: Test Welghts for K-Basin

$570 \mathrm{~kJ}$ TEST WETHT

DRY WEGHT: 1439.72 LBS

UNDERWATER WEGHT: 1256.62 LBS

SURFACE AREA 1861.64 $\mathrm{IN}^{\wedge} 2$

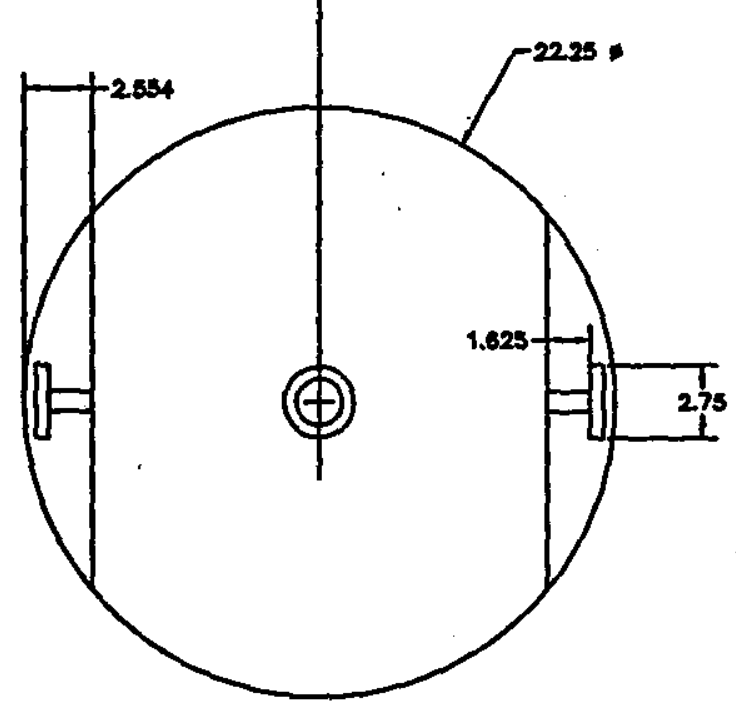

Page A9

FIGURE A2 - $570 \mathrm{~kg}$ SOLID 304 SST TEST WEIGHT 
FLUOR DANIEL NORTHWEST, INC.

\section{CALCULATIONS AND SKETCHES SHEET}

\begin{tabular}{|c|c|c|c|}
\hline DEPARTMENT: & $\begin{array}{l}\text { Mechanisms } \\
\text { Engineering }\end{array}$ & ORIGINATEO BY: & $5 / 20 / 99$ \\
\hline ENG COMM NO: & & $l_{\text {lad }} k$ DATE: & $5 / 20 / 9$ \\
\hline AREA: & $100 \mathrm{~K}$ & REVISED BY: & \\
\hline SUBJECT: & \multicolumn{3}{|c|}{ Test Weights for K-Basin } \\
\hline
\end{tabular}

Numbers Based on Solid Model Development using AutoCAD R14

Test Welght Work Sheet - Solid Steel

\begin{tabular}{|c|c|c|}
\hline $\begin{array}{l}304 \text { SST } \\
\text { Water @ 50 F }\end{array}$ & $\begin{array}{l}7.900 \\
0.9997\end{array}$ & $\begin{array}{l}0.285 \\
0.036\end{array}$ \\
\hline
\end{tabular}

- Source CRC Handbook of Chemistry and Physics, 77th Edition

\section{Lfiting Attachment Volume and Weight Calcs}

Consists of $0.75^{\prime \prime}$ OD Rod and $0.50^{\prime \prime}$ Plate (304 SST)

\begin{tabular}{|c|c|c|c|c|c|}
\hline Rod & $\begin{array}{c}\text { Dia (in) } \\
0.750\end{array}$ & $\begin{array}{l}\text { Length (In) } \\
1.625\end{array}$ & $\begin{array}{c}\text { Vol (lin^3) } \\
0.718\end{array}$ & $\begin{array}{c}\text { (DRY) } \\
\text { Lb } \\
0.205\end{array}$ & \\
\hline & $\begin{array}{c}\text { Length (in) } \\
2.750\end{array}$ & $\begin{array}{c}\text { Wjdth (in) } \\
3.630\end{array}$ & $\begin{array}{c}\text { Thick (in) } \\
0.500\end{array}$ & $\begin{array}{c}\text { Vol (in^3) } \\
4.991\end{array}$ & $\begin{array}{c}\mathrm{Lb} \\
1.422\end{array}$ \\
\hline
\end{tabular}

Total Volume: 5.709 in^3

Total Welght: 1.627 lbs

Flgure A2 - Development of $570 \mathrm{~kg}$ Welght

\begin{tabular}{|c|c|c|c|c|c|c|c|}
\hline $\begin{array}{l}\text { Helght is } 11.875^{\prime \prime} \\
\text { Steel Shell with cutout } \\
\text { Displaced Water }\end{array}$ & $\begin{array}{c}\ln ^{\wedge} 3 \\
\text { Main } \\
4485.047 \\
4510.265\end{array}$ & $\begin{array}{l}\ln ^{\wedge} \mathbf{3} \\
\text { Plate } \\
0.000\end{array}$ & $\begin{array}{l}\ln ^{\wedge} 3 \\
\text { Lugs } \\
11.418 \\
11.418\end{array}$ & $\begin{array}{c}\ln ^{\wedge} 3 \\
\text { Total } \\
4496.465 \\
4521.683\end{array}$ & $\begin{array}{l}\text { Total } \rightarrow \\
\text { Total } \rightarrow\end{array}$ & $\begin{array}{c}\text { Wt lbs } \\
1281.492 \\
-162.781 \\
1281.492 \\
1118.712\end{array}$ & $\begin{array}{l}\text { Dry } \\
\text { Wet }\end{array}$ \\
\hline $\begin{array}{l}\text { Check Weight } \\
\text { Change per inch }\end{array}$ & $\begin{array}{l}137.928 \\
109.236\end{array}$ & $\begin{array}{l}\text { Ib Under } \\
\mathrm{lb} / \mathrm{in}\end{array}$ & & & & & \\
\hline $\begin{array}{l}\text { Estimate add } \\
\text { New Height }\end{array}$ & $\begin{array}{r}1.25 \\
13.12\end{array}$ & & & & & & \\
\hline \multicolumn{3}{|c|}{ Check New Height of $13.125^{\prime \prime}$} & & & & & \\
\hline Steel Shell with cutout & $\begin{array}{c}\text { in^3 } \\
\text { Main } \\
4964.229\end{array}$ & $\begin{array}{l}\text { In^3 } \\
\text { Plate }\end{array}$ & $\begin{array}{l}\text { in^3 } \\
\text { Lugs } \\
11.418\end{array}$ & $\underset{\substack{\text { In^3 } \\
\text { Total } \\
4975.647}}{4}$ & & $\begin{array}{l}\text { Wt lbs } \\
1418.059\end{array}$ & \\
\hline
\end{tabular}


FLUOR DANIEL NORTHWEST, INC.

CALCULATIONS AND SKETCHES SHEET

\begin{tabular}{|c|c|c|c|}
\hline DEPARTMENT: & $\begin{array}{l}\text { Mechanisms } \\
\text { Engineering }\end{array}$ & ORIGINATED BY: H.t Roagh & DATE: $5 / 20 / 59$ \\
\hline ENG COMM NO: & & 11 llenback & DATE: $5 / 2$ \\
\hline AREA: & $100 \mathrm{~K}$ & REVISED BY: & DATE: \\
\hline
\end{tabular}

SUBJECT: Test Welghts for K-Basin

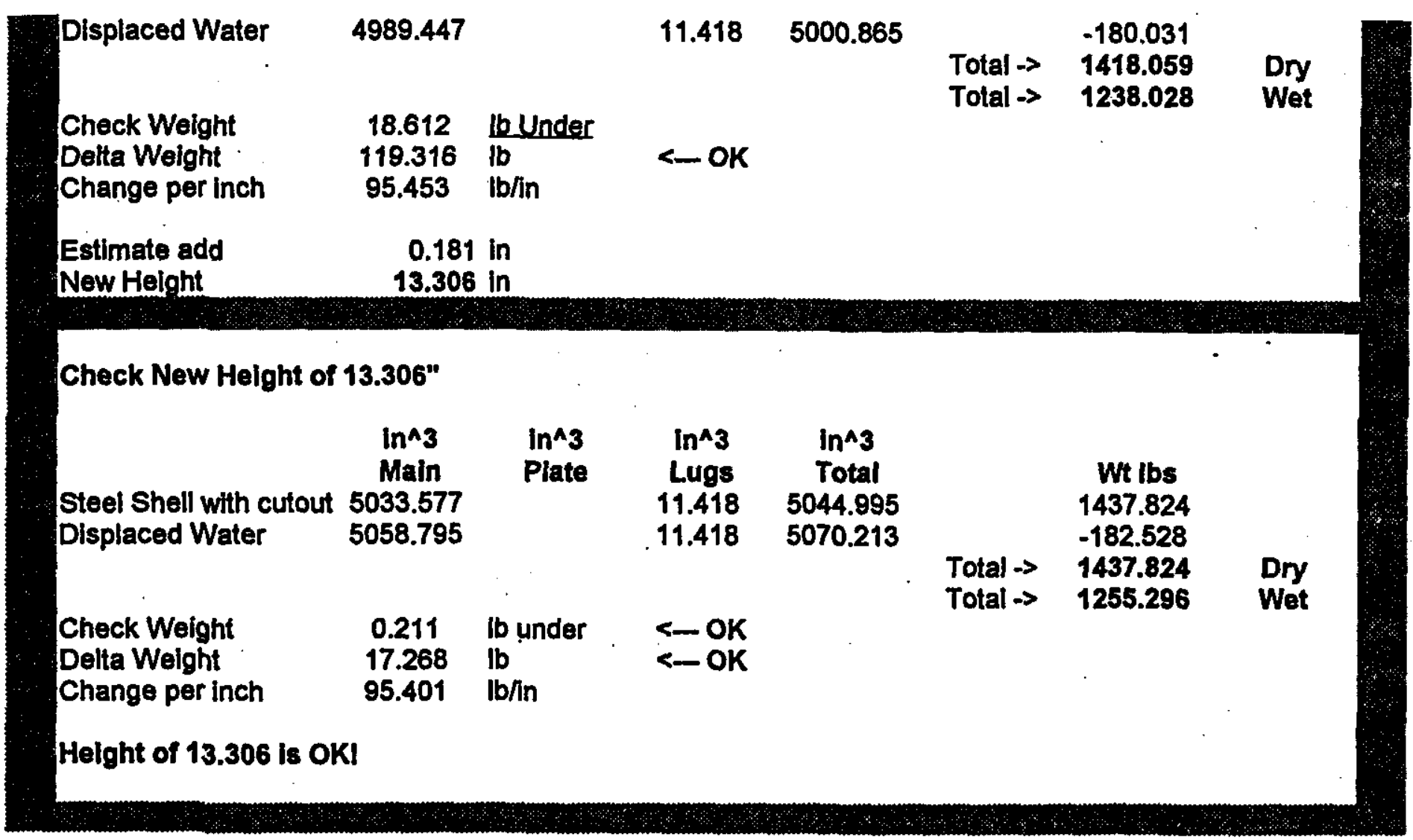


FLUOR DANIEL NORTHWEST, INC.

HNF-4460, / Rev 1

CALCULATIONS AND SKETCHES SHEET

\begin{tabular}{|c|c|c|c|}
\hline DEPARTMENT: & $\begin{array}{l}\text { Mechanisms } \\
\text { Engineering }\end{array}$ & ORIGINATED BY: & DATE: $5 / 20 / 59$ \\
\hline ENG COMM NO: & & $=C K E D$ & DATE: \\
\hline
\end{tabular}

SUBJECT: Test Weights for K-Basin

2.3 Conceptual Design and Physical Properties of Lifting Beam for Test Weights

Figure A3 shows a conceptual design for a lifting beam designed to allow the test weight sections to be lowered into the K-basin.

Construction is all 304 Stainless Steel. Load capacity is $1800 \mathrm{lbs}$. 
FLUOR DANIEL NORTHWEST, INC.

HNF-4460, Rev 1

CALCULATIONS AND SKETCHES SHEET

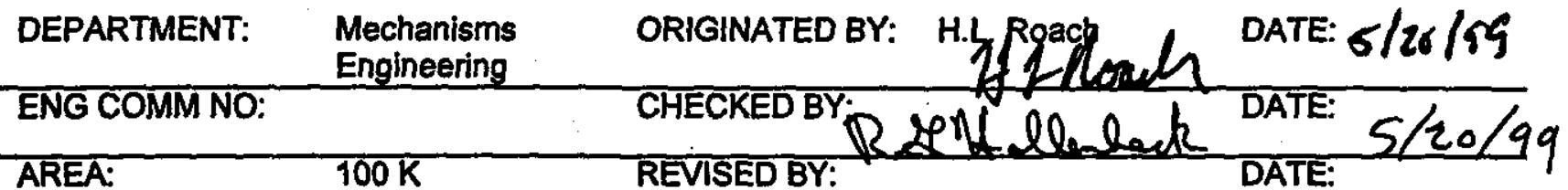

SUBJECT: Test WoIghts for K-Basin

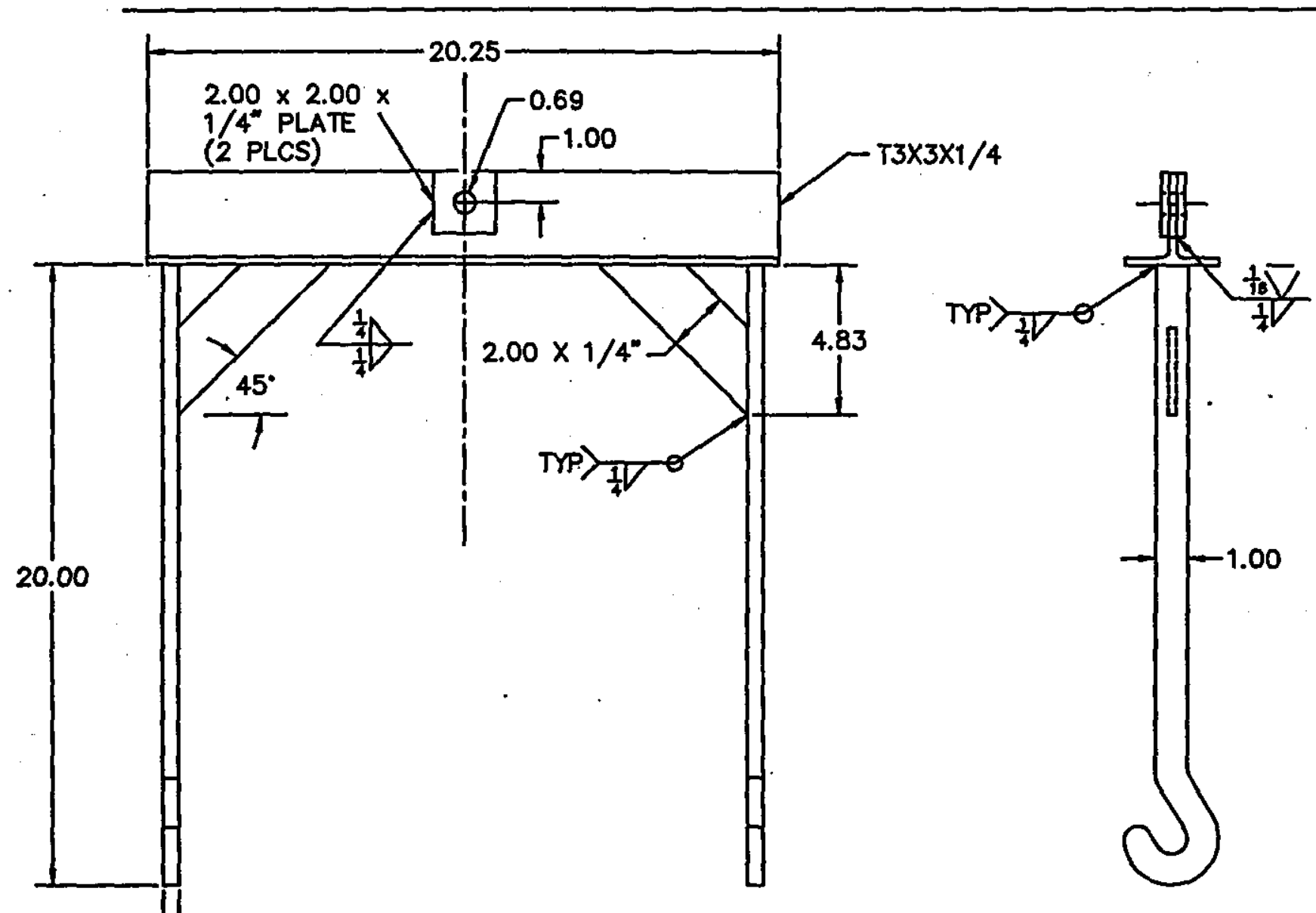

FIGURE A3 - IEST WEIGHT SPREADER BEAM

1800 LB LOAD CAPACITY

ALL 304 STAINLESS STEEL

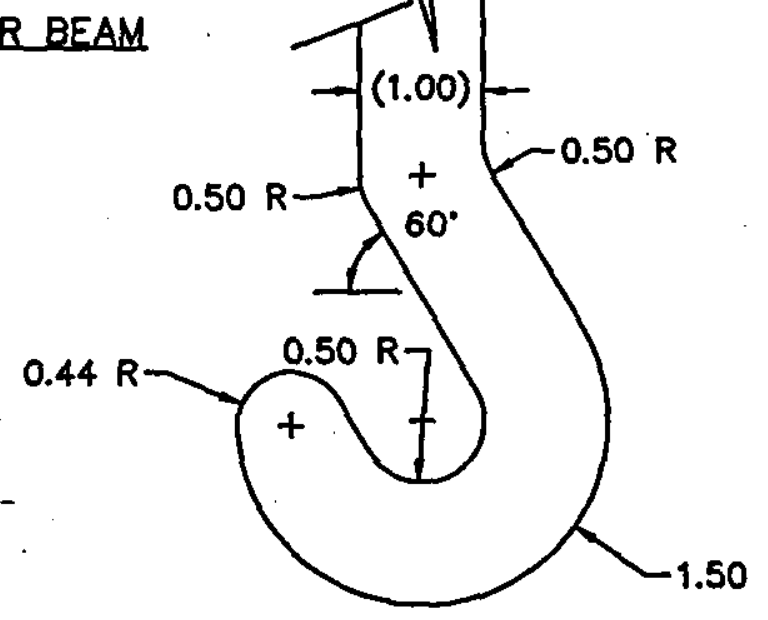

HOOK DEIAIL 


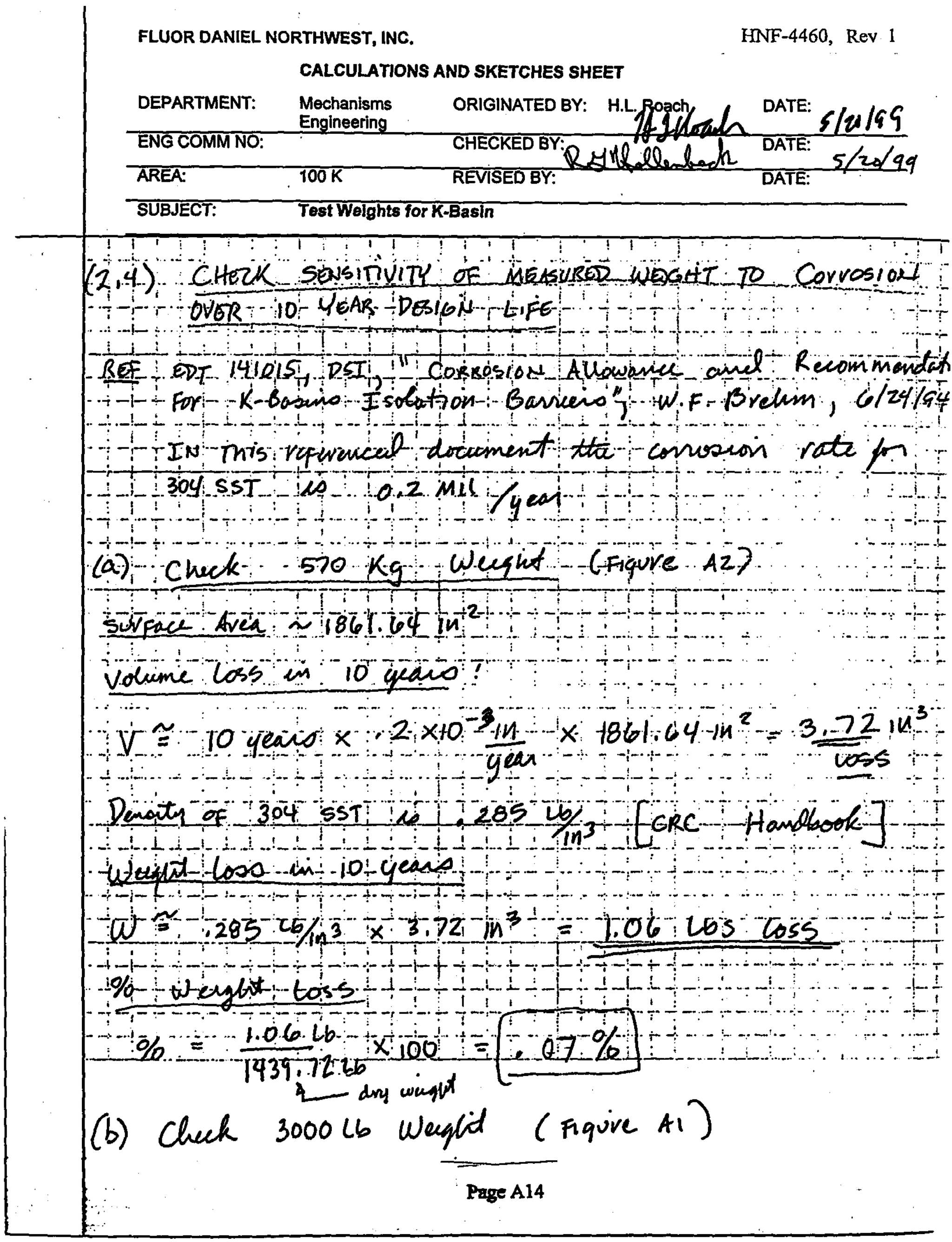




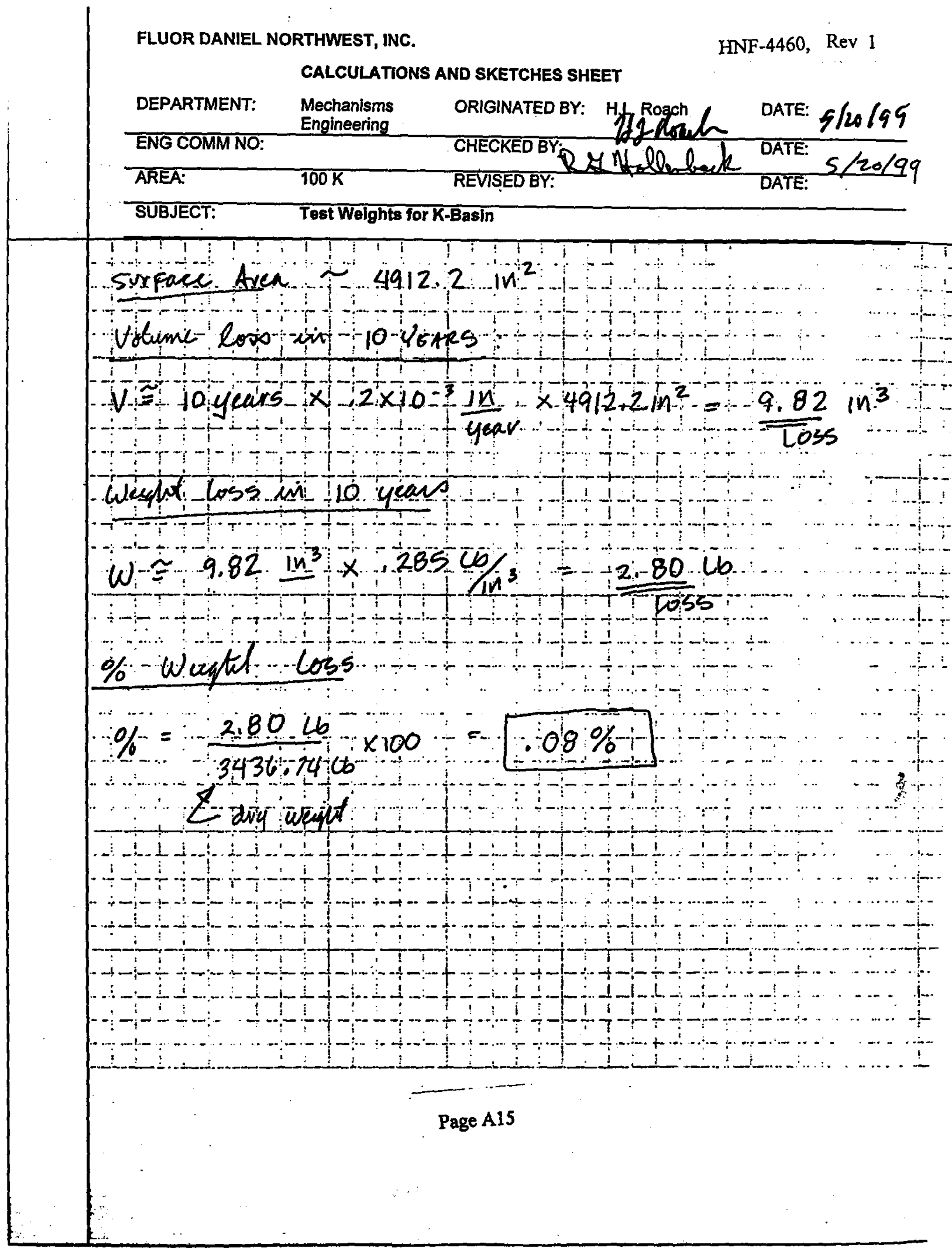




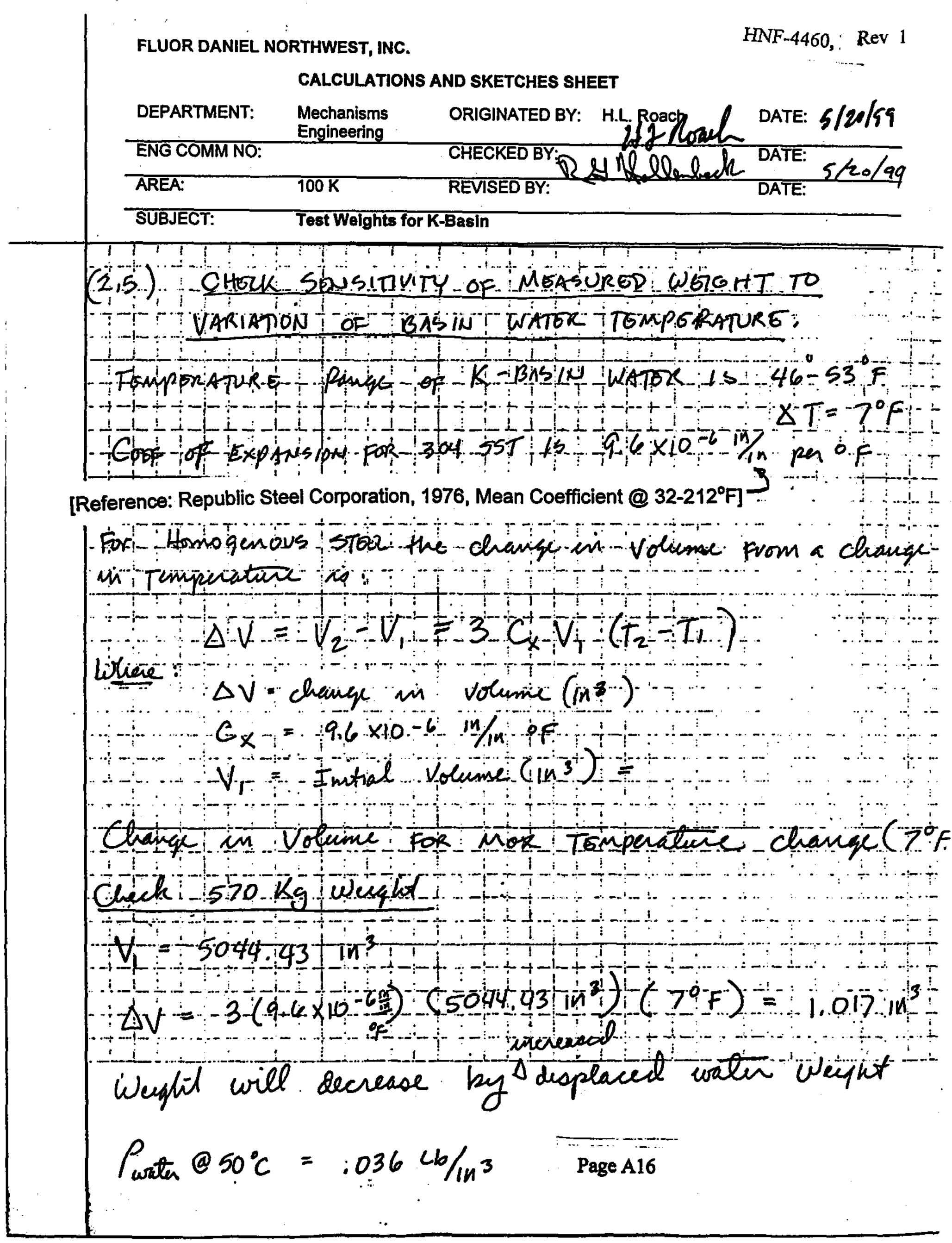




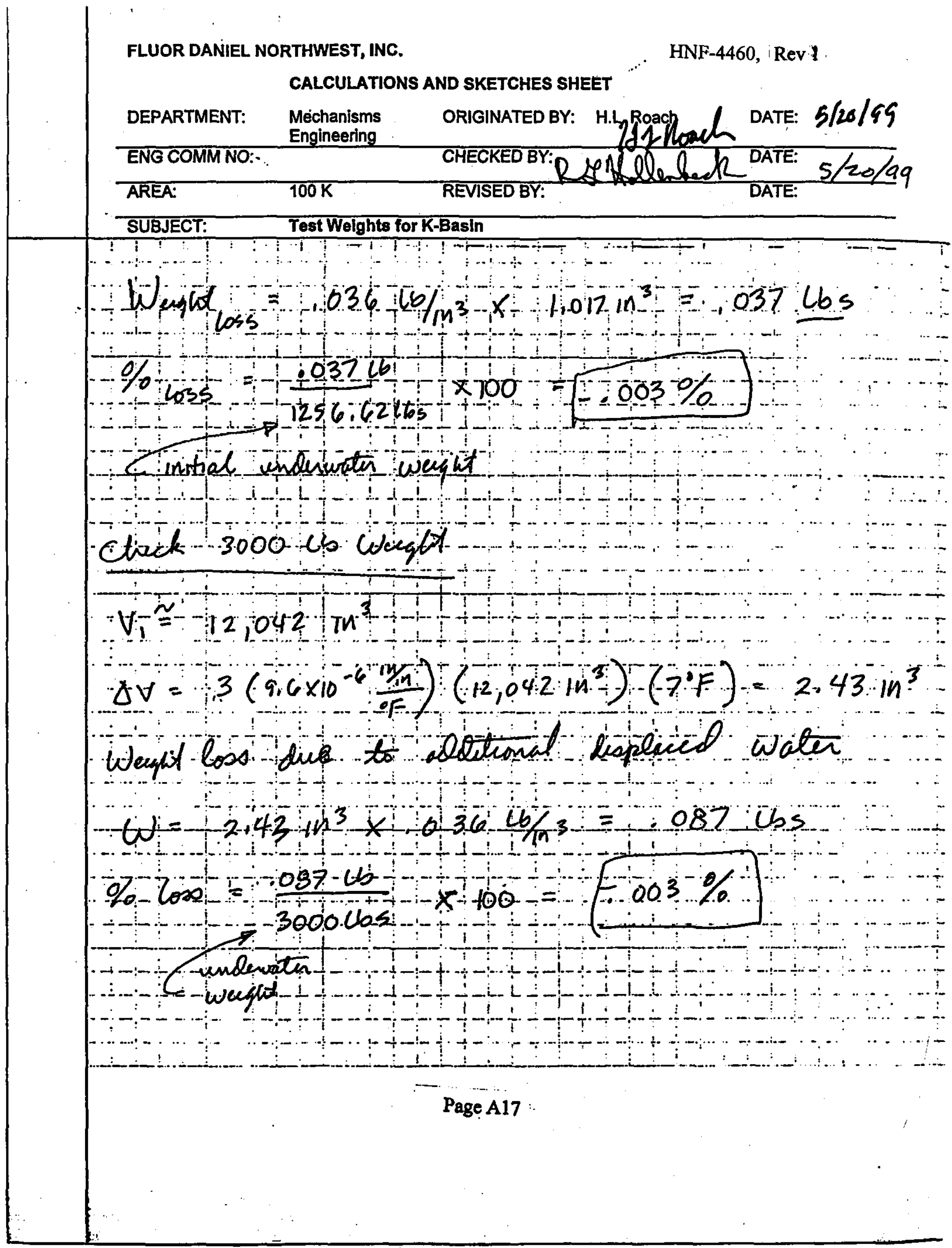




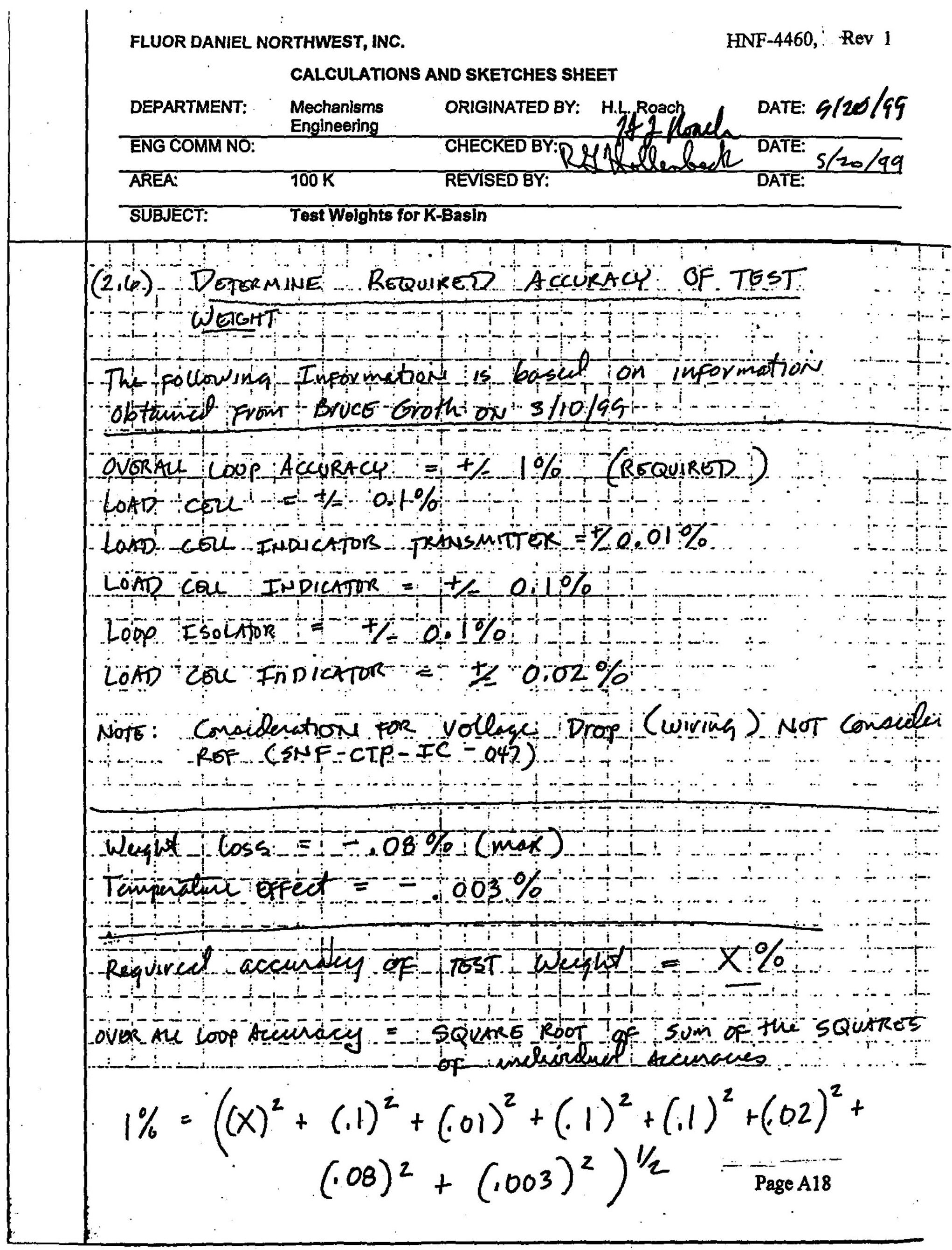




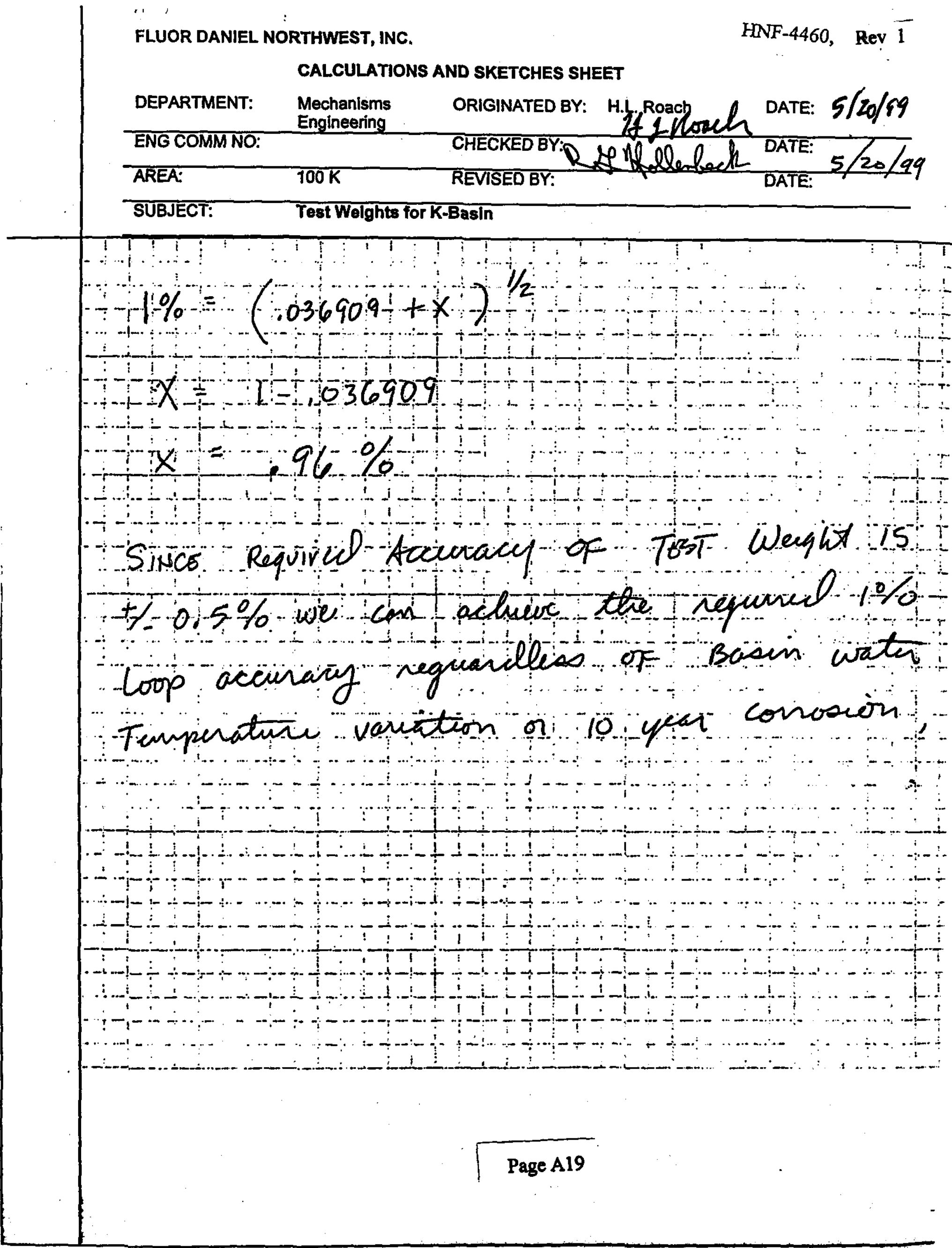




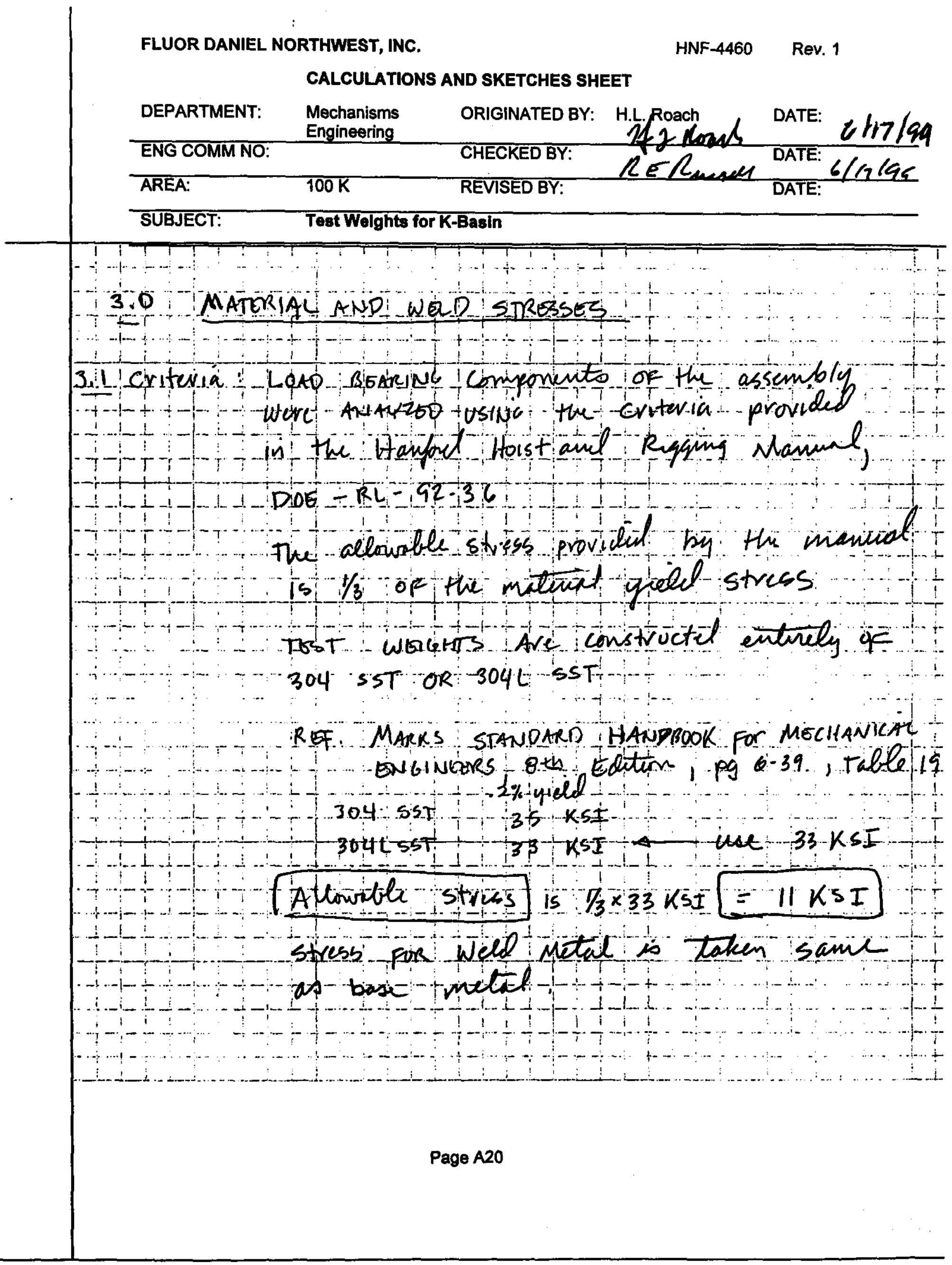




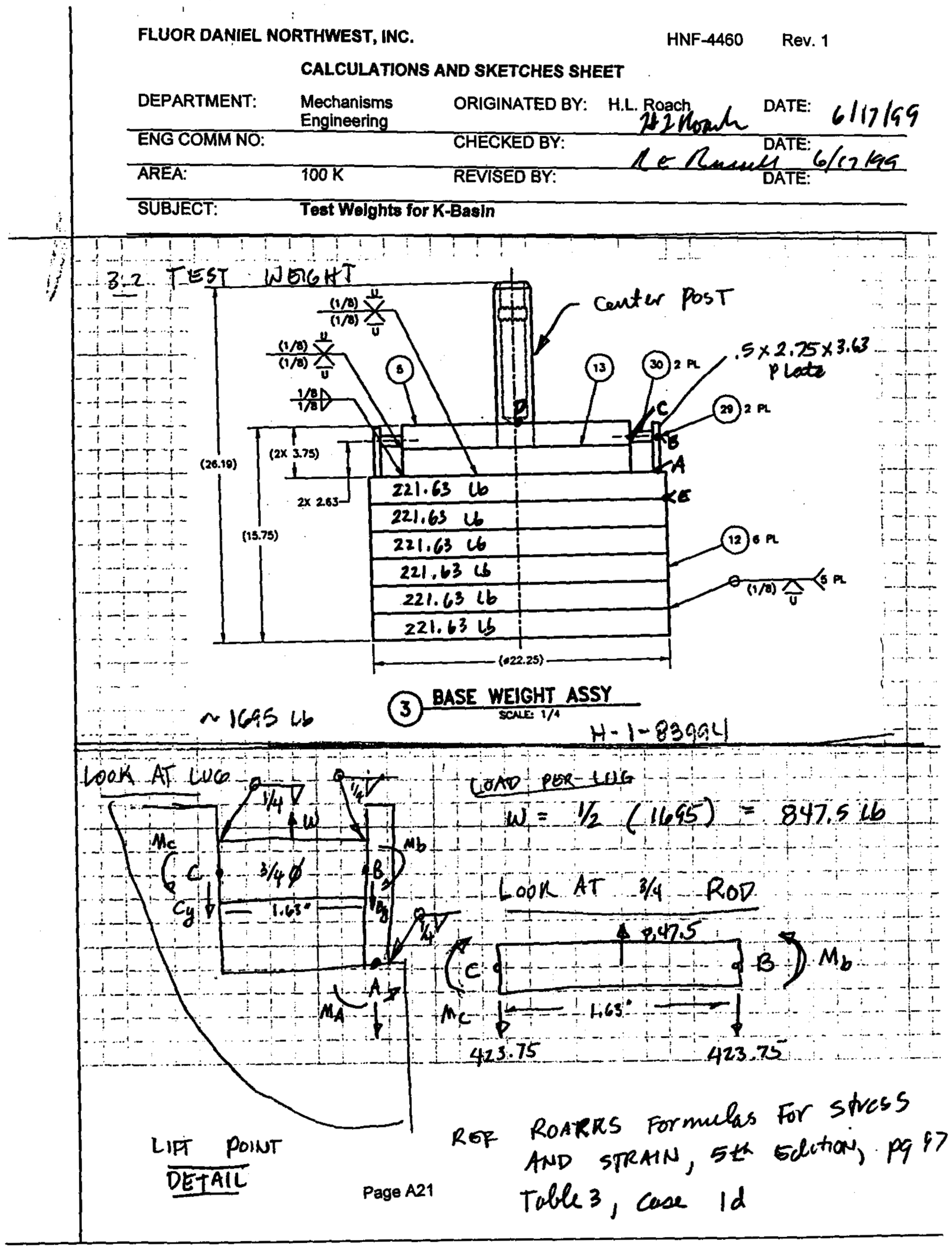




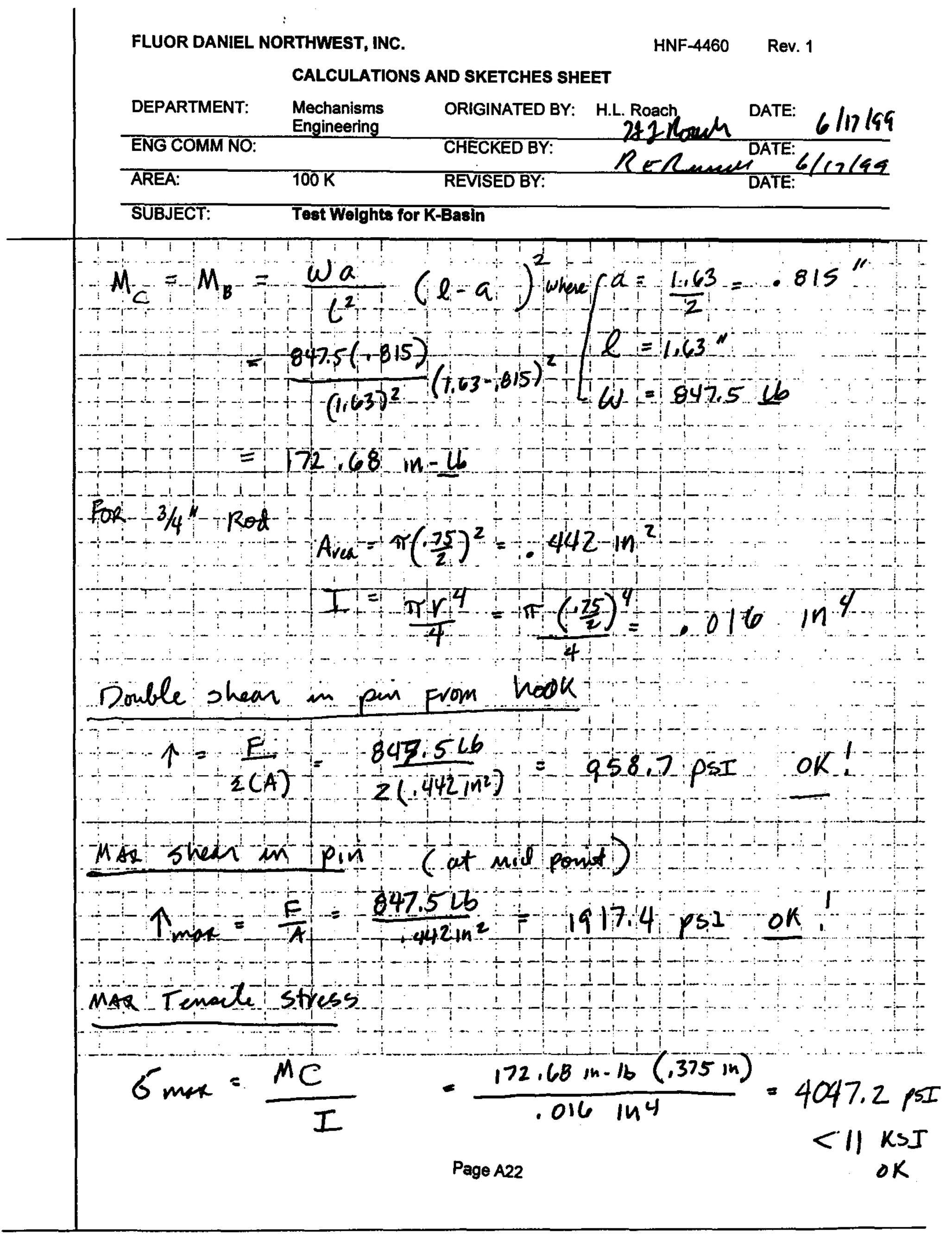




$$
\begin{aligned}
& \text { FLUOR DANIEL NORTHWEST, INC. } \\
& \text { HNF-4460 Rev. } 1 \\
& \text { CALCULATIONS AND SKETCHES SHEET }
\end{aligned}
$$

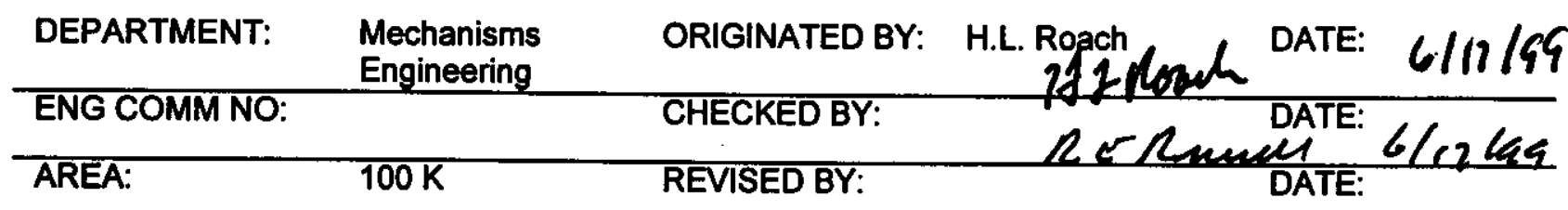

$$
\begin{aligned}
& \text { SUBJECT: } \quad \text { Test Weights for K-Basin } \\
& \text { Pin weir stresses } \\
& \text { Fill }=\operatorname{sill} A=H, 000(707 \omega)=7777 \omega\left(\frac{\mathrm{L}}{\mathrm{m}}\right)
\end{aligned}
$$

Force per inch of well

$$
f_{b}=\frac{M}{2 \omega}=\frac{132.68 m-16}{4421 n^{2}}=390.68 \frac{16}{1 n}
$$

Vertices shea

$$
f_{5}=\frac{V}{L w}=\frac{423.7546}{\pi(75)}=179.854 / 5
$$$$
\text { length }
$$

Resultant $f \equiv\left(390.68^{2}+779.85^{2}\right) 1 / 2=430.09 \mathrm{lg} / 1 \mathrm{n}$

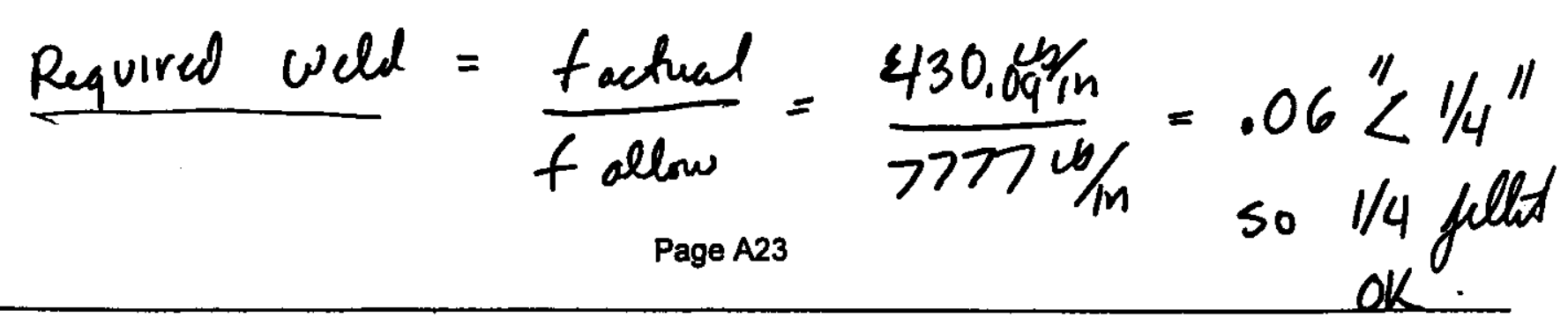




$$
\begin{aligned}
& \text { FLUOR DANIEL NORTHWEST, INC. } \\
& \text { iNF }-4460 \\
& \text { Rev. } 1 \\
& \text { CALCULATIONS AND SKETCHES SHEET }
\end{aligned}
$$

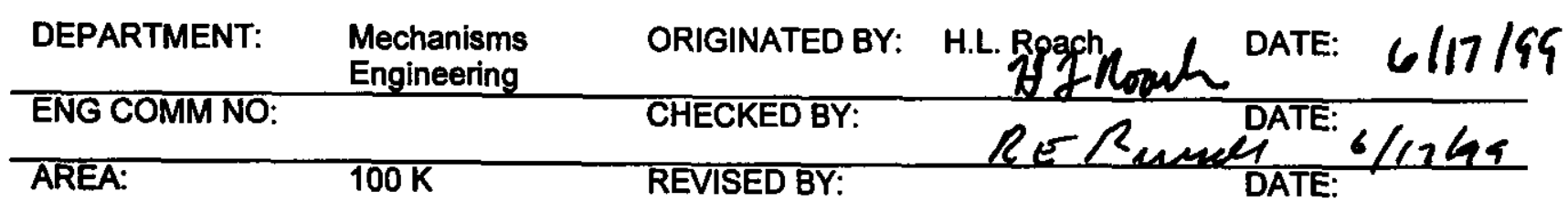

$$
\begin{aligned}
& \text { SUBJECT: Test Weights for K-Basin } \\
& M_{b}=172.66 \text { in } 15 \\
& \text { Therefore } F_{a}=F_{b}=423,7526 \\
& M_{A}-M_{b}=72.66 \mathrm{in}-16 \\
& 2.75=b \quad .5=d \\
& =(.5)(2.75)+\frac{(65)^{2}}{3}=4.4 \mathrm{in}^{2}
\end{aligned}
$$

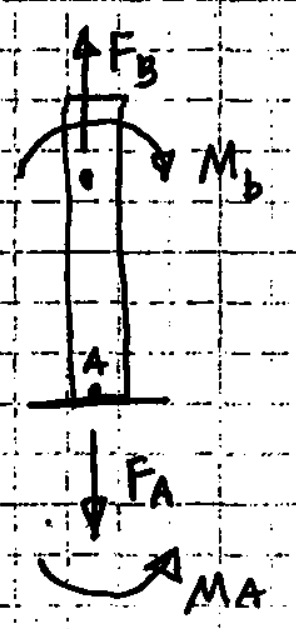

From benisons Moment

$$
f=\frac{M}{z_{w}}=\frac{72+68 \ln 16}{-1+6 \ln ^{2}}=1+8 \cdot 3 \frac{16}{1 n}
$$

From tension

$$
f_{t}=\frac{p}{L_{w}}=\frac{423,751 b}{(2,75)(2)+(.5)(2)}=65,2 \frac{\mathrm{kb}}{11}
$$

Resultant

$$
\begin{aligned}
f_{R}=\left(f_{b}{ }^{2}+f_{t}^{2}\right)^{1 / 2} & =\left(118.3^{2}+65.2^{2}\right)^{1 / 2} \\
& =135.1 \frac{16}{\mathrm{in}}
\end{aligned}
$$




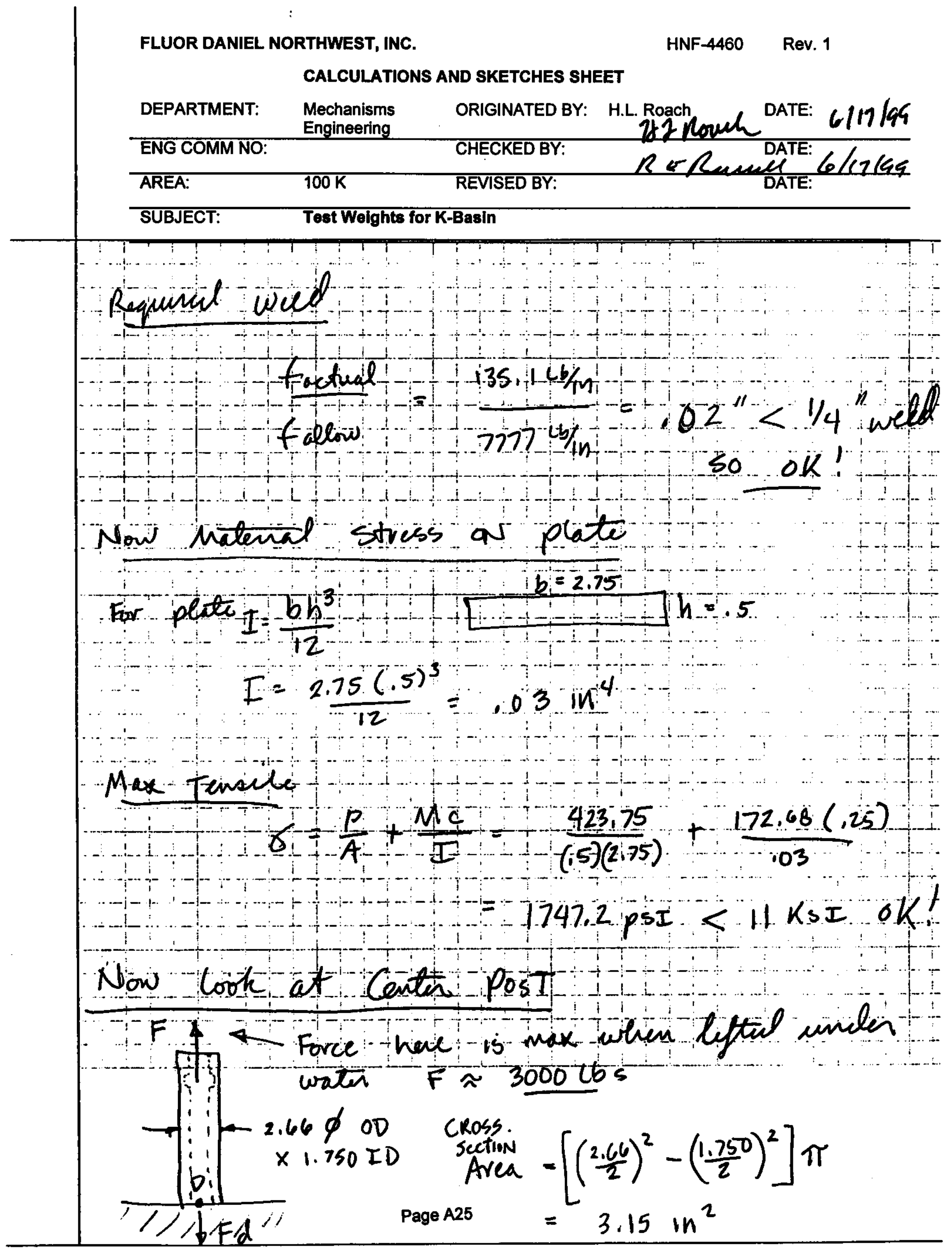




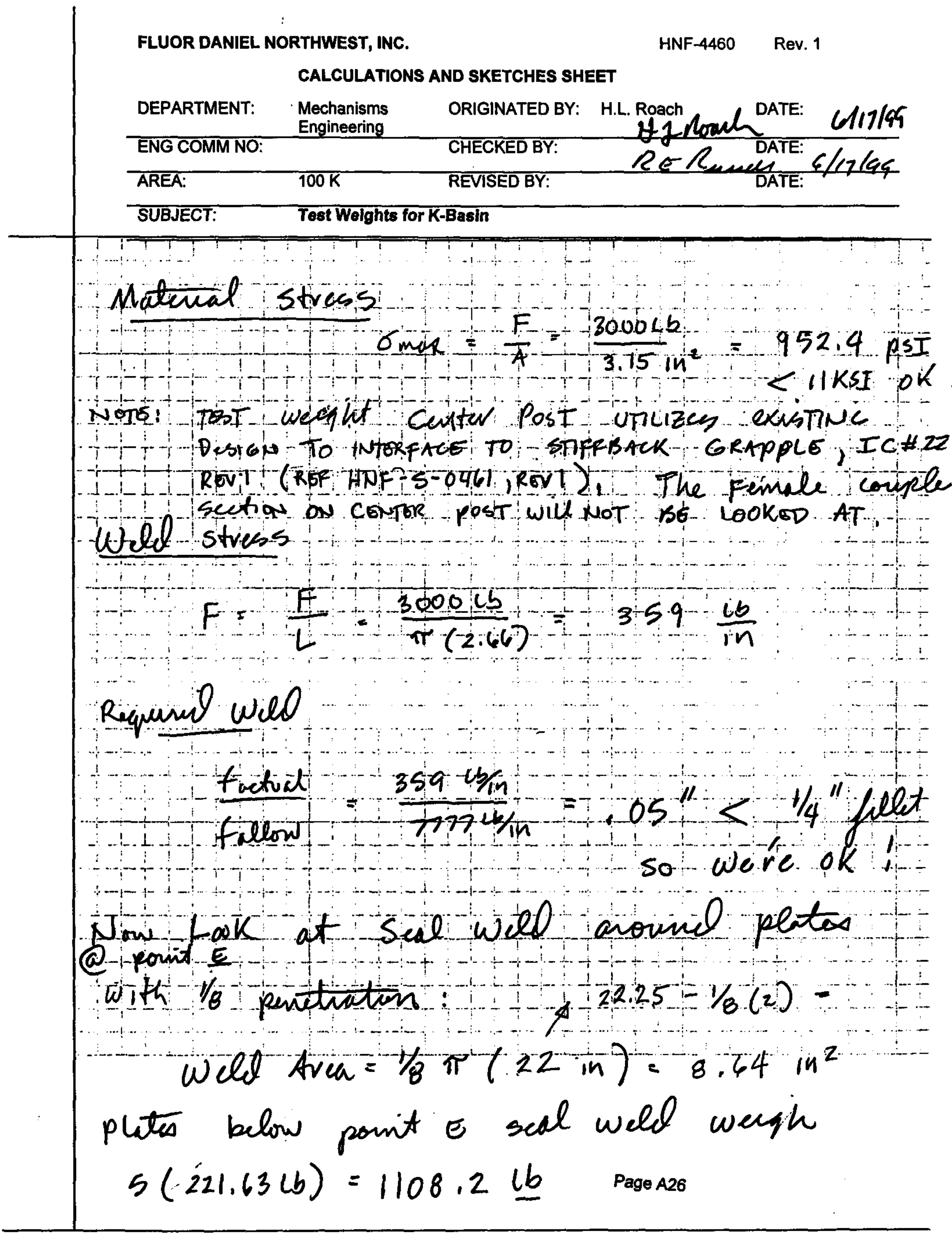




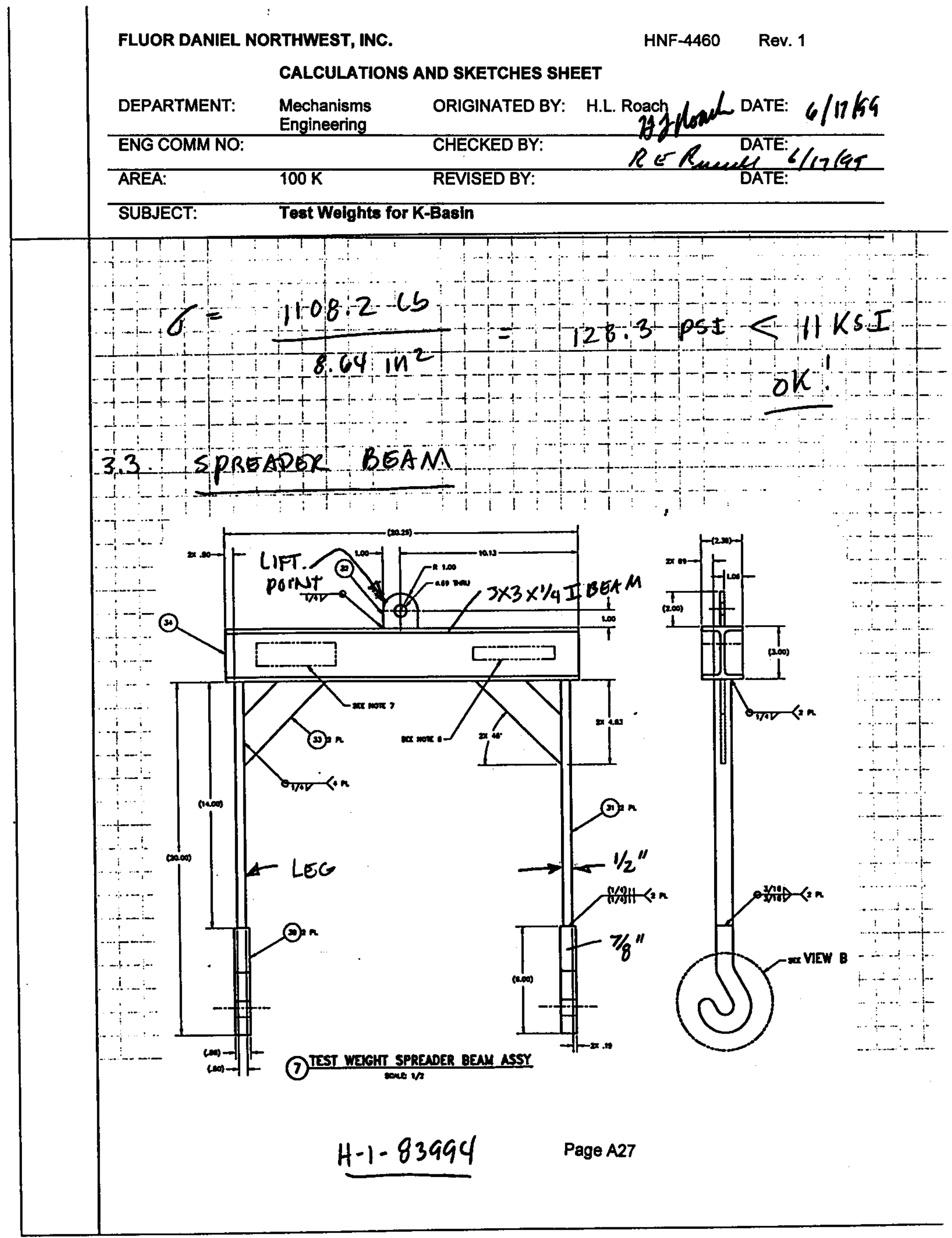




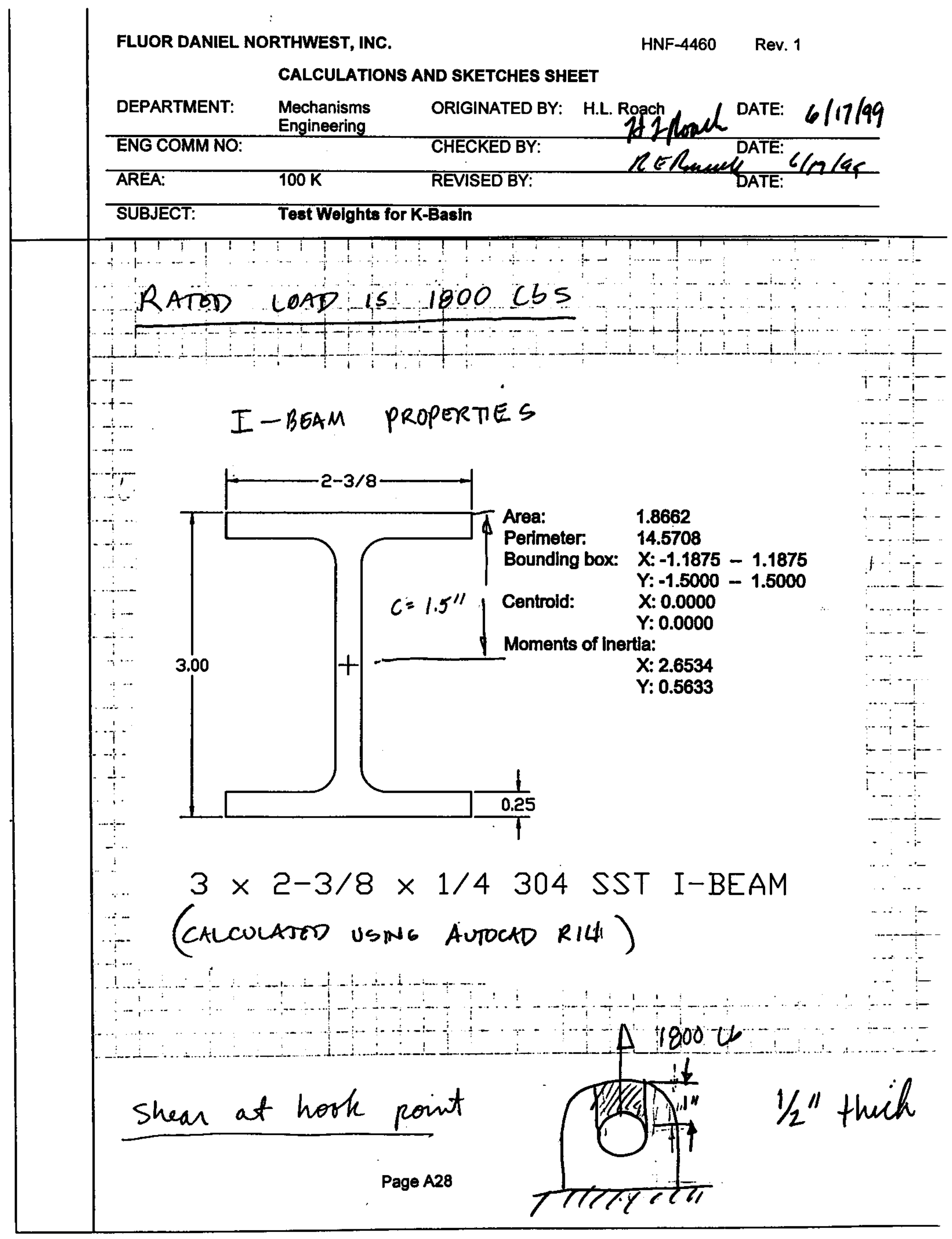




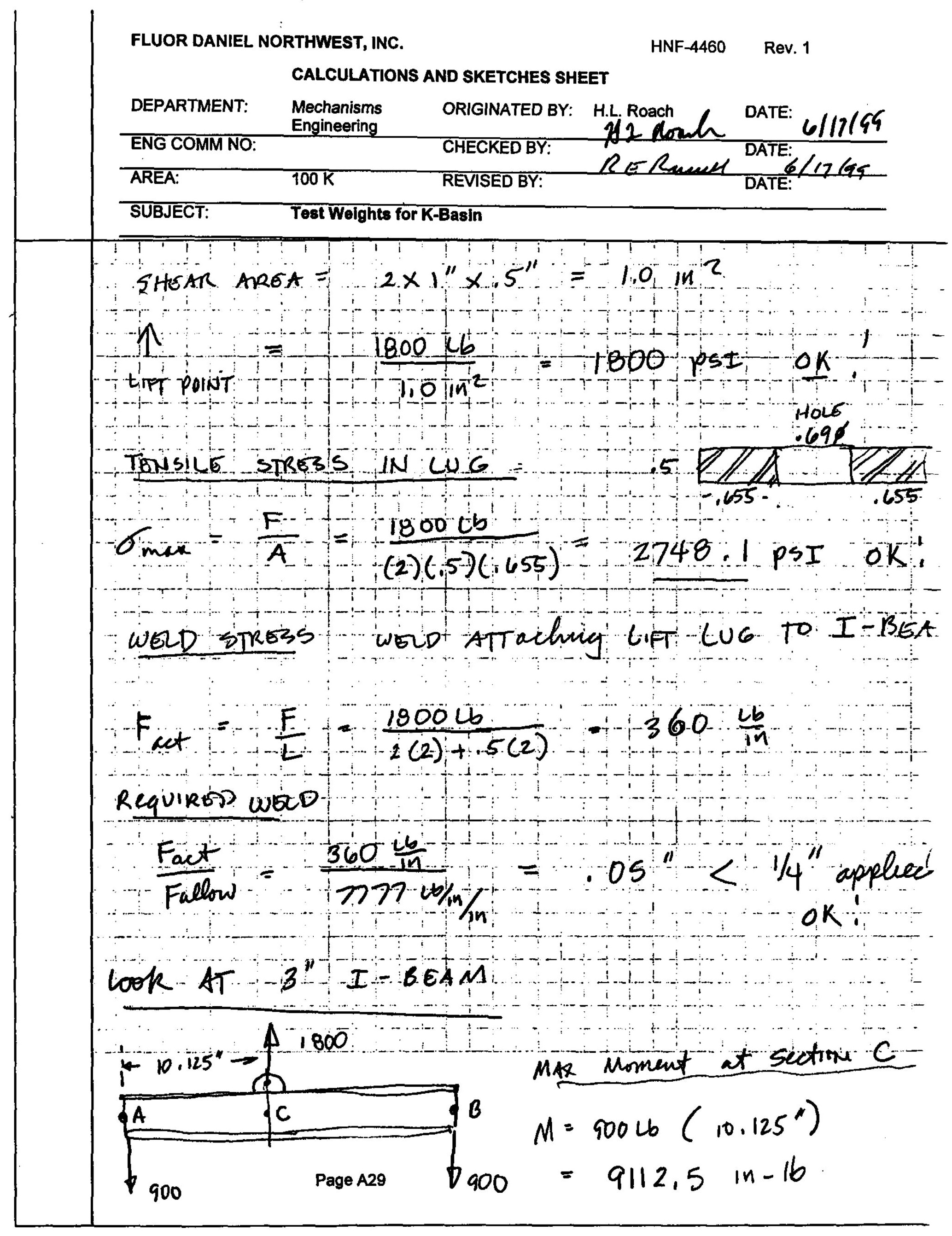




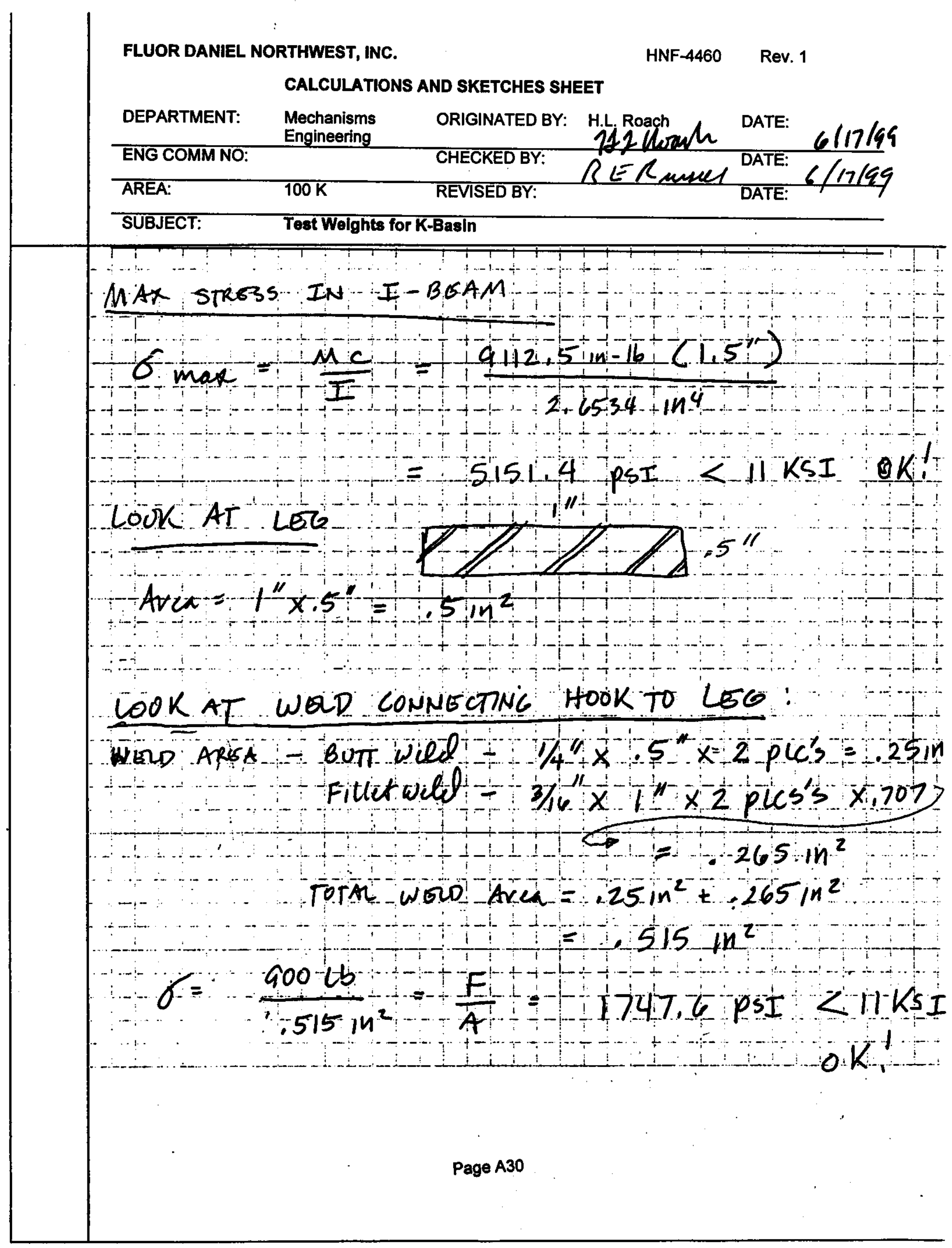




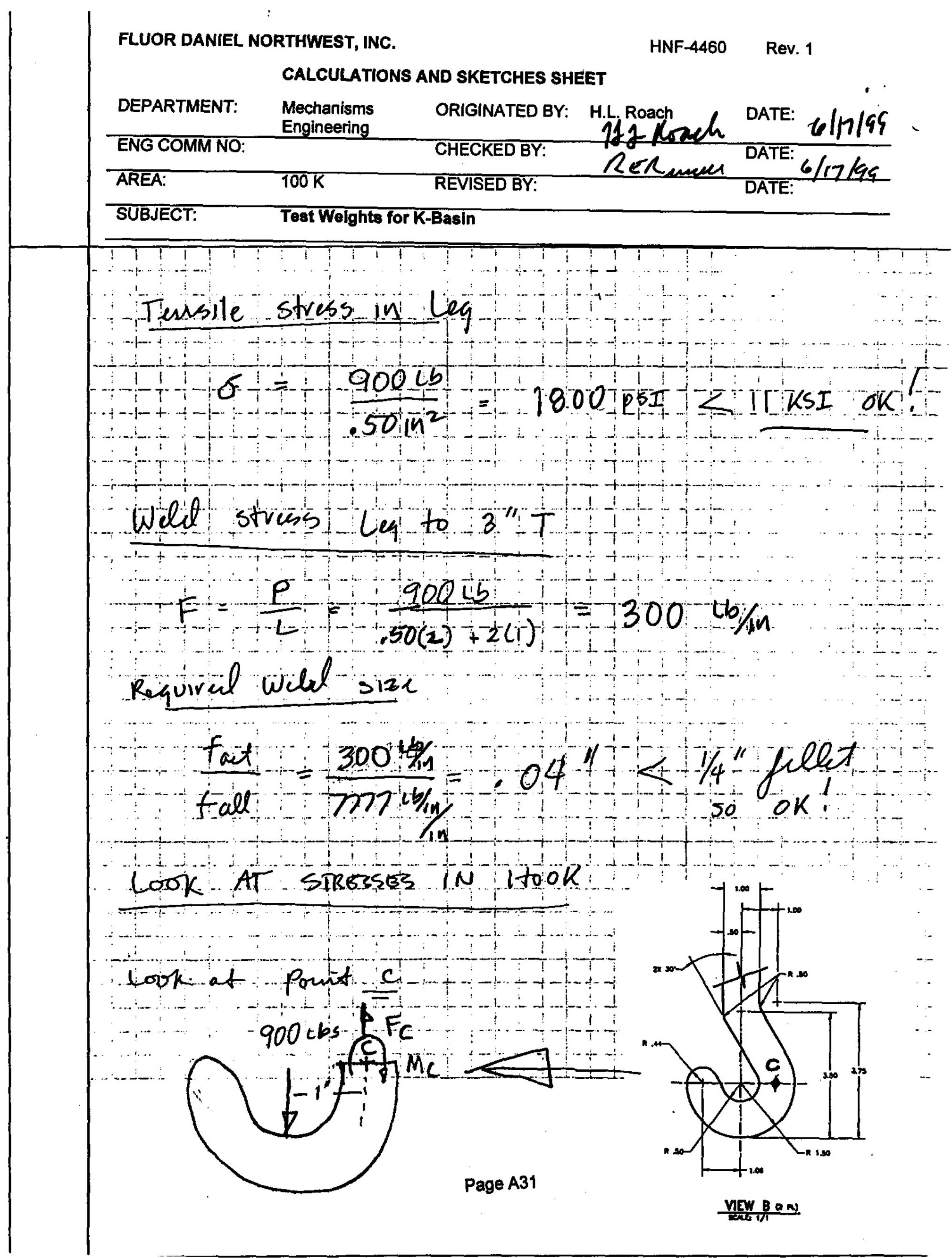




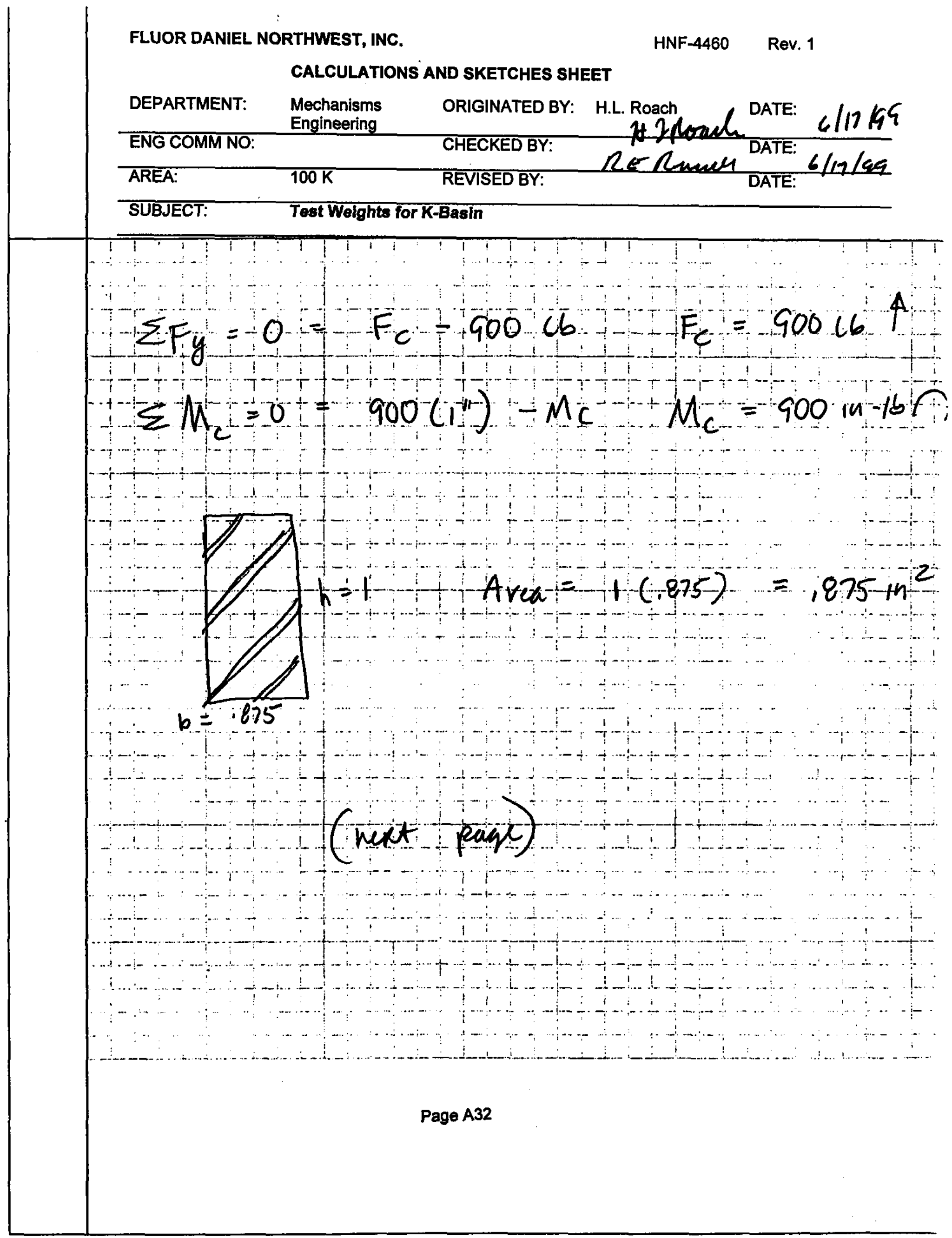




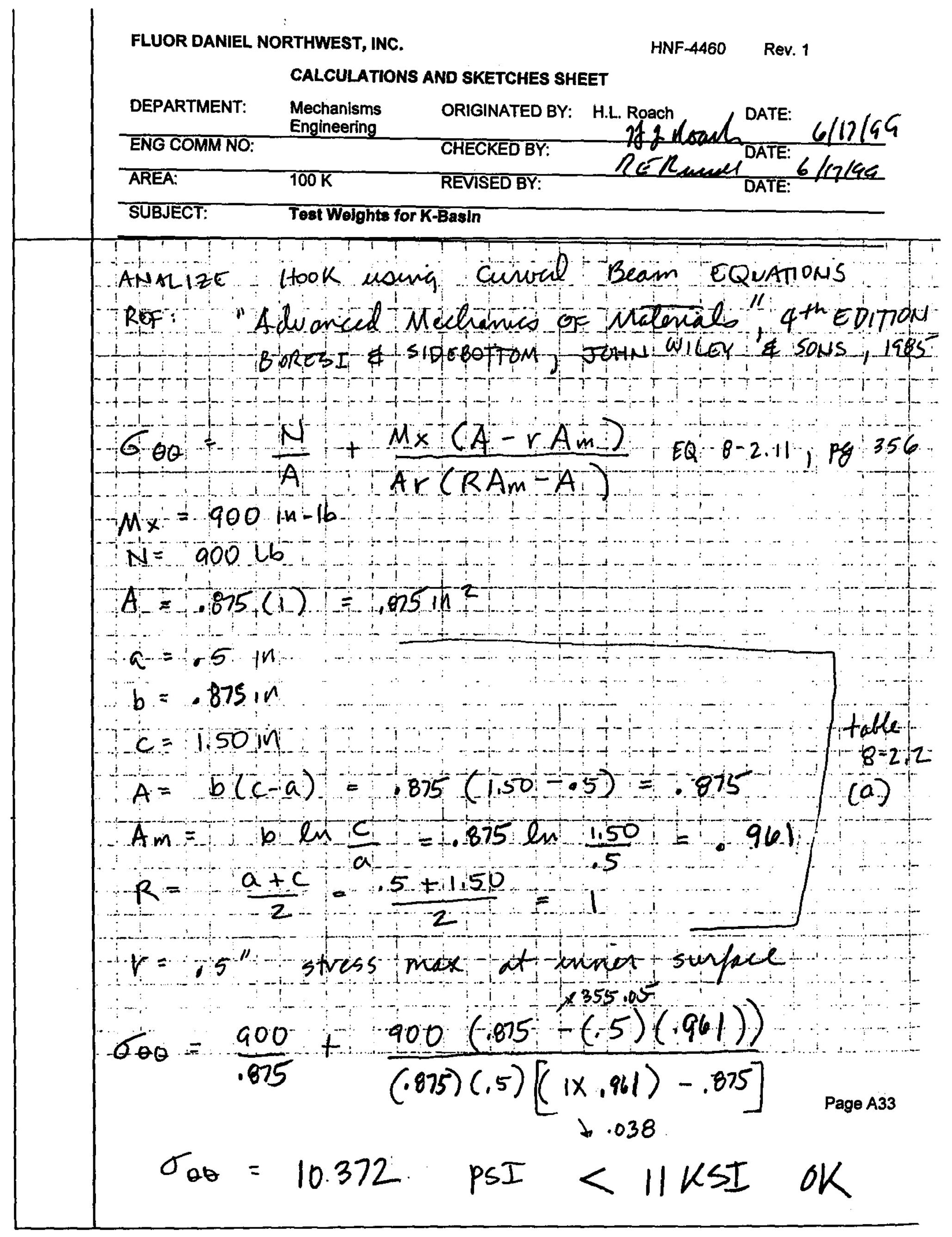


Fuel Retrieval System Small Tools Design Package

Test Weights

HNF-4460, Rev 1

August 25, 1999

Page $B-0$

Appendix B

Test Weight Test/Calibration 


\section{ORIGINAL}

\section{ENEROY \\ NORTHWEST \\ Standards Laboratory}

Plant Support Facility

MD 1025, PO Box 968

Richland, WA 99353-0968

Phone (509) 377-8131 FAX (509) 377-8219

\section{Certificate of Calibration}

Manufacturer: MSI

Description: DYNAMOMETER

Asset Number: 545-29-06-046

Report Number: 936278220

Customer: BOYD JA1
Model: $\mathbf{7 2 0 0}$

Serial Number: 66669

Ref. Number: 75

\section{CALIBRATION INFORMATION}

\section{Test Conditions:}

Calibration Date: 2-Sep-99

Calibration Due: 2-Sep-00

Procedure / Rev: SLI 30-11 REV. 1

Technician: $\quad$ E. L. SHARP

Remarks: $\quad$ TESTED WTH TYPE "F" WEIGHT SETS. NOTE: "F" CLASS

WEIGHTS ARE 0.01\%-RATIO OF STDS 10:1 MIN.

Test Results:

$\begin{array}{ll}\text { Pass: } & \mathbf{Y} \\ \text { Incomplete: } & \mathrm{N} \\ \text { Limited: } & \mathrm{N} \\ \text { As Found: } & \text { Pass } \\ \text { As Left: } & \text { Pass }\end{array}$

\section{STANDARDS USED FOR CALIBRATION}

\begin{tabular}{|c|c|c|c|c|c|}
\hline Asset Number & Manuf & Model & Description & Calibration Date & Due Date \\
\hline 0059369 & RICE LAKE & N/A & WEIGHT SET & 5-Aug-99 & 5-Aug-00 \\
\hline 44420 & AKO & N/A & WEIGHT SET & 11-Mar-99 & 11-Mar-00 \\
\hline
\end{tabular}

Notes/General Conditions:

The standards and calibration program of the Energy Northwest Standands Laboratory complies with the requirements of 10 CFR50 Appendix B and ANSI/NCSL Z-540-1.

- Unless otherwise noted:

The standards used in this calibration, described in the referenced calibration procedure with associated uncertainties or tolerances, are traceable to the National Institute of Standards and Technology (NIST). There are no special limitations of use imposed on this item. This Report may not be reprodyced, except in full, without the permission of the Energy Northwest Standards Laboratory.

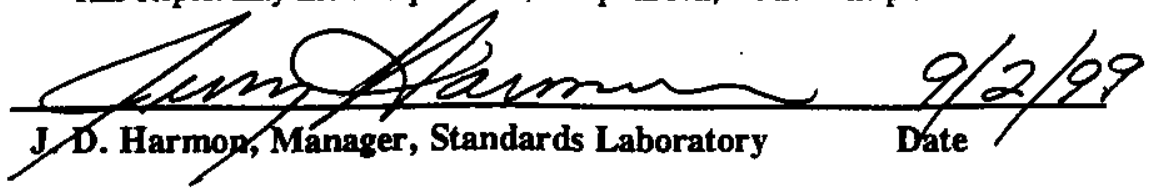




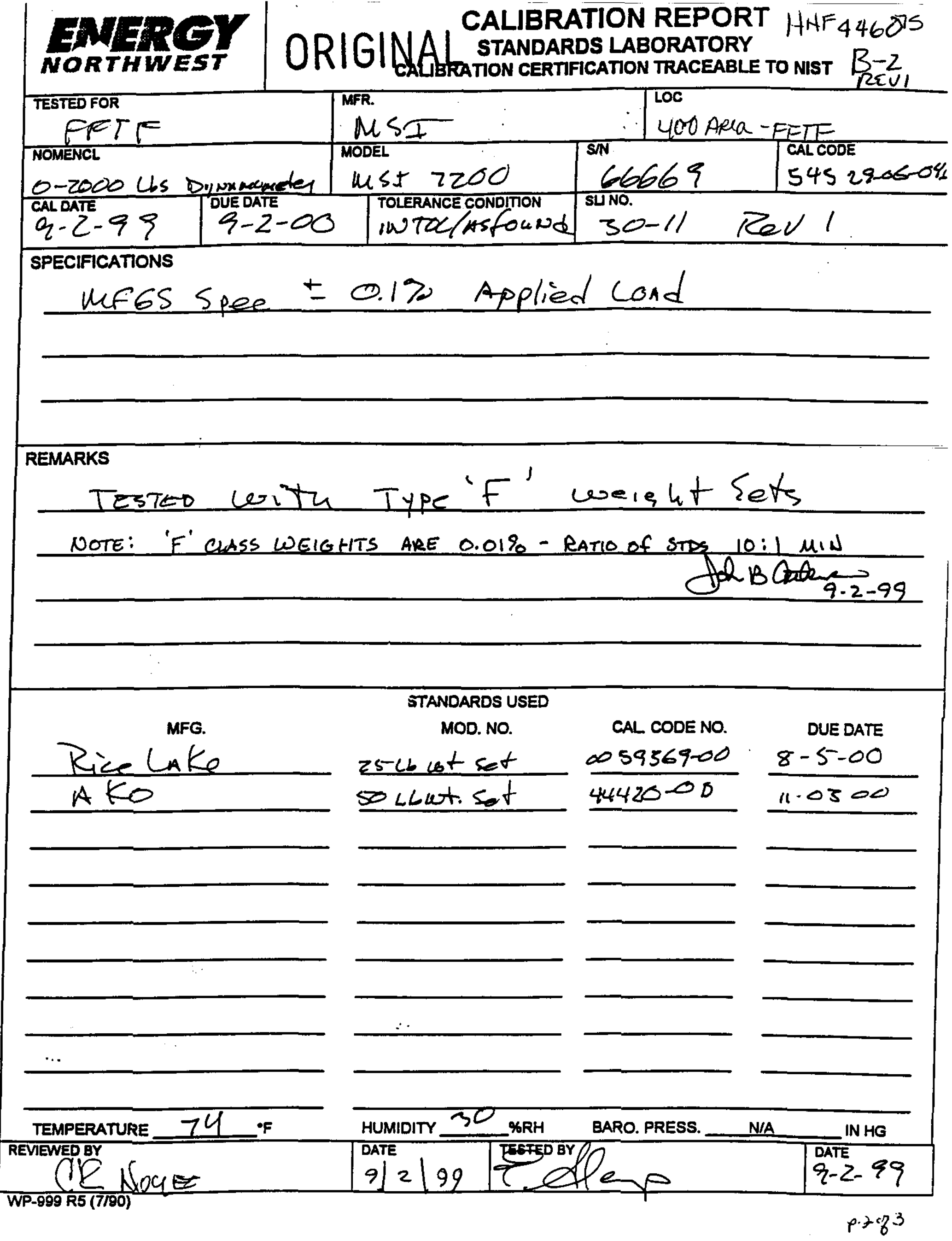




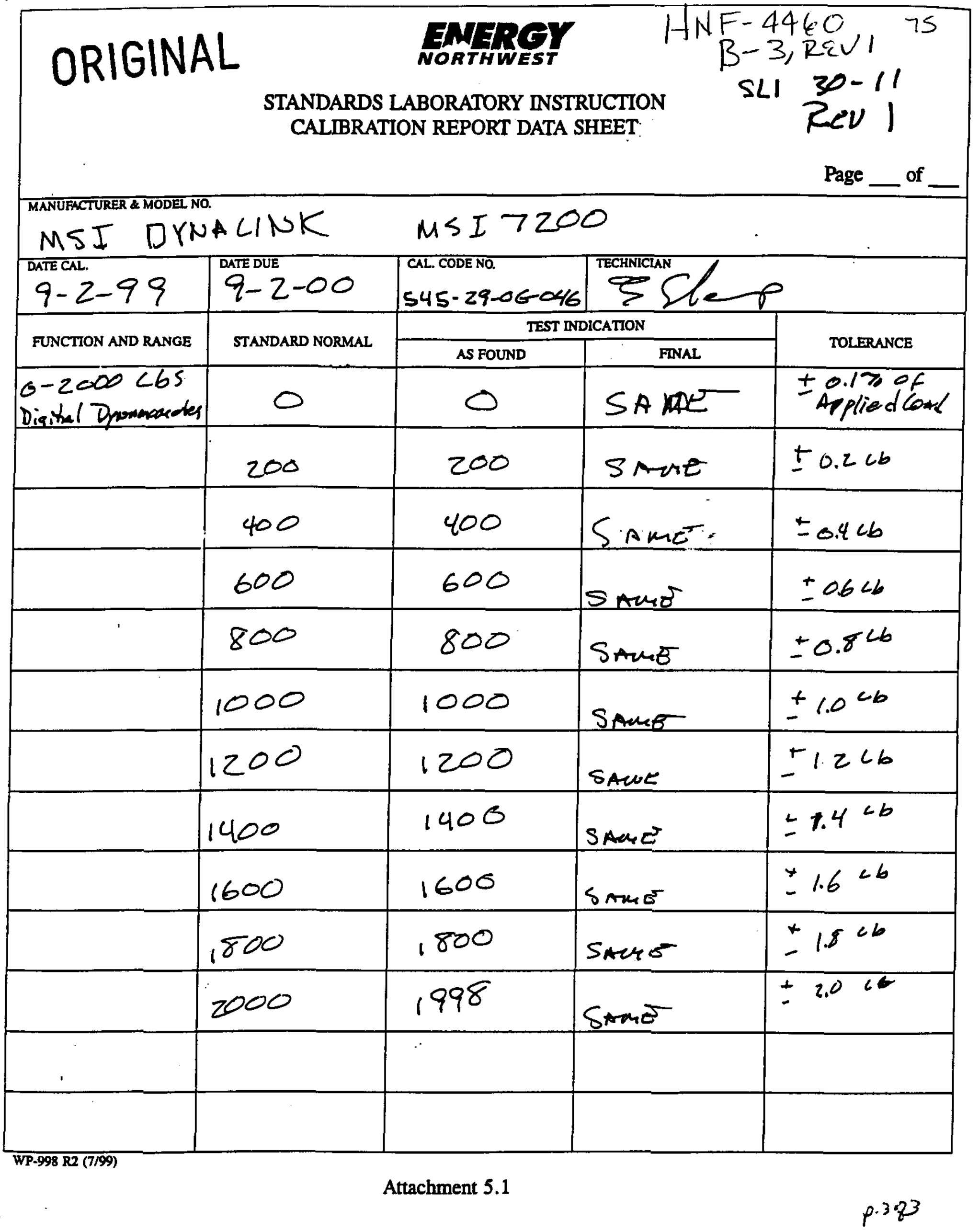




\section{ORIGINAL}

\section{EMEROY \\ NORTHWEST \\ Standards Laboratory}

Plant Support Facility

MD 1025, PO Box 968

Richland, WA 99353-0968

Phone (509) 377-8131 FAX (509) 377-8219

\section{Certificate of Calibration}

\begin{tabular}{rlrl} 
Manufacturer: & SHOPMADE & Model: & N/A \\
Description: & STAINLESS STEEL WEIGHT & \\
Asset Number: & $999-86-02-103$ & Serial Number: & H-1-83994-040 \\
Report Number: & 935663100 & Ref. Number: & 75 \\
Customer: & STECKER SO & & \\
\hline
\end{tabular}

CALIBRATION INFORMATION

Test Conditions:

Calibration Date: 26-Aug-99

Temperature: $0.0 \mathrm{C}$

Calibration Due: 26-Aug-00

Humidity: $0 \%$

Procedure / Rev: SLI 30-12 REV.0

Technician: W. E. CALLAWAY

Remarks: $\quad$ CALIBRATED IN 305BLDG-USED AT K-BASIN

\section{Test Results:}

$\begin{array}{ll}\text { Pass: } & \mathbf{Y} \\ \text { Incomplete: } & \mathbf{N} \\ \text { Limited: } & \mathbf{N} \\ \text { As Found: } & \text { Pass } \\ \text { As I } \text { eft: } & \text { Pass }\end{array}$

\section{STANDARDS USED FOR CALIBRATION}

\section{Asset Number}

\section{Asset Number}

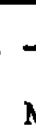

MSI
Model

7200
Description

DYNAMOMETER
Calibration Date Due Date

21-Jan-99

\section{Notes/General Conditions:}

The standards and calibration program of the Energy Northwest Standards Laboratory complies with the requirements of 10 CFR50 Appendix B and ANSI/NCSL Z-540-1.

Unless otherwise noted:

The standards used in this calibration, described in the referenced calibration procedure with associated uncertainties or tolerances, are traceable to the National Institure of Standards and Technology (NIST). There are no special limitations of use imposed on this item. This Report may not be reproduced, except in full, withoux the permission of the Energy Northwest Standards Laboratory.

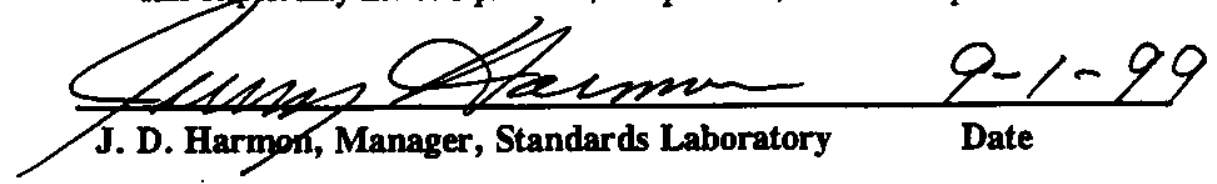




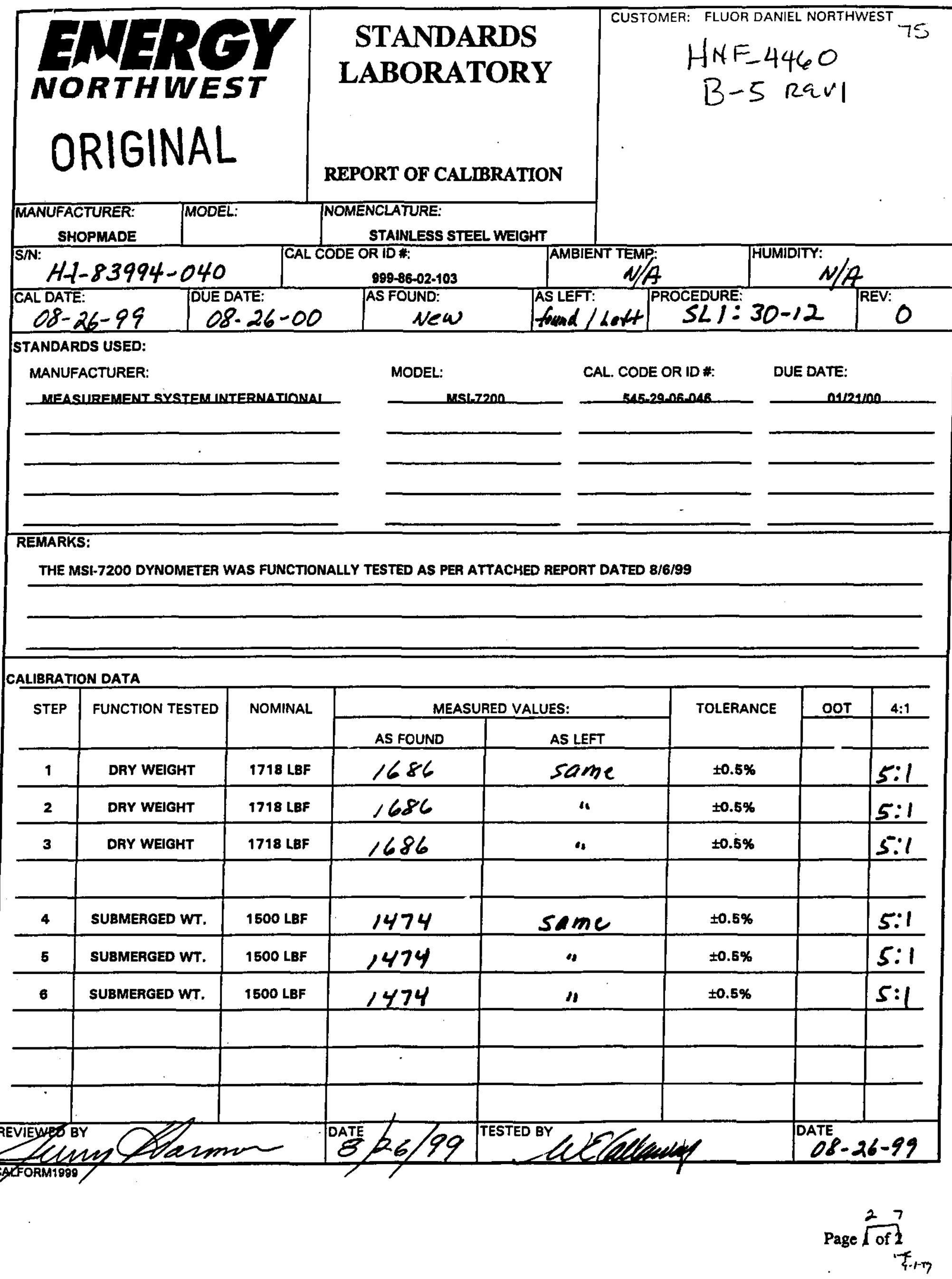




\section{ORIGINAL}

$999-840+103$

$$
\begin{gathered}
\text { HNF }-4460 \\
B=5 \\
\text { REV }
\end{gathered}
$$

\section{FLUOR DANIEL}

Fluor Daniel Hanford, Inc.

P.O. Box 1000

Richland, WA 99352

\section{RROJECT EANEORD (PH)}

Contract:

Release : 00075

rxecuted: 08/13/99

Printed : 08/13/99

Paga :

Mil Involes To:

Fluox Daniel Banford

ATHN: ACCOUNTS RAYART: 61-80

Po Box 1000

RICBIAND WA 99352
Vendor:

Michael I. Milson

WASEINGTON FUEIC POWER SUPPLY PO BOX 968/MS-055

RICEIAND WA 99352

Pleace Direct Inquirios to:

Fork Location:

STEVEAY O. STFCLER C.P.M.

Title: CONIRACTING OETICER

Phone: 509-373-7715

Ext:

Fax : 509-376-9016

TitI0: IRS - CAIIBRATION OF TWST FEICATS FOR K-BASIAYS - JNGRGY NW

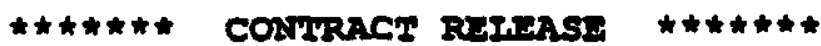

Total value : $\$ 5,000.00$ USD

Pricing Method: Estraptis

Contract Type : SHRVICAS

Project

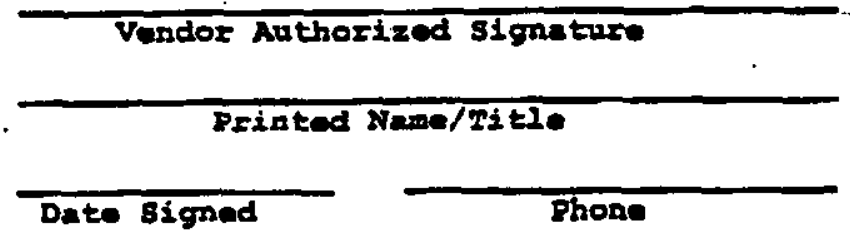

* NOT TO EXCHa **.

Start Date: 07/26/99

End Date : 09/30/99

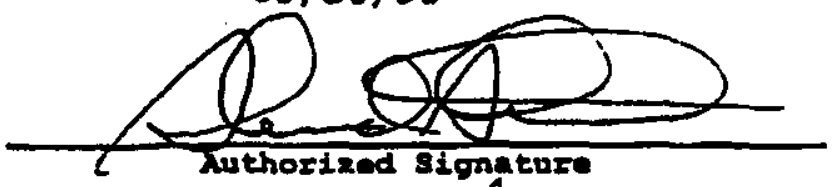

STEUTN O STeden Pristed Namo/Titlo

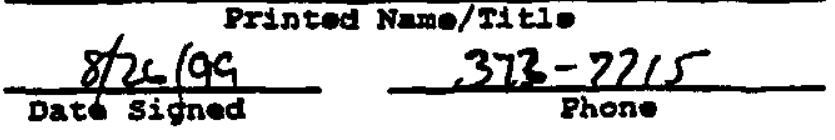




\section{STATEMENT OF WORK FOR \\ Callbrtwoution of Test Weights for $\mathbf{K}-$ Basin}

\subsection{OBJECTIVE}

This action is to provide for the certification of two test weights traceable to the National Institute of Standards Testing (NIST) standards. The test weights will be used at the $K$ Basins to perform a daily calibration of the weighing system on the monorail mounted Multi-Canister Overpack (MCO) basket stiffback grapple.

All Hanford Site Measurement and Test Equipment (M\&TE) calibration/certification work is subcontracted by DynCorp to Energy NW. The certification of the test weight will be done by Energy NW.

\section{$2.0 \quad$ INTRODUCTION}

The Spent Nuclear Fuel (SNF) Project has been established to provide facilities and systems with which to retrieve, repackage, and interim store the spent nuclear fuel from the $\mathrm{K}$ Basins in a safe configuration away from the Columbia River. The Fuel Retrieval System (FRS) Sub-project has been established to design, install, and test a system to repackage the spent nuclear fuel in the $K$ Basins. Fuel retrieval is accomplished using underwater process equipment. The SNF is loaded into MCO baskets and the MCO baskets are transferred into a MCO using the MCO basket stifiback grappie. The MCO basket stiffback grapple bas an integral weighing system that is used to weigh the loaded MCO baskets. Test weights will be used to perform a daily accuracy and linearity check of the MCO basket stiffback grapple weighing system.

Two test weights have been designed to be used with the MCO basket stiffback grapple to provide the required daily linearity check of the weighing system.

The $3000 \mathrm{lb}$. test weight assembly, H-1-83994; Item 1, is designed to provide approximate 3000 lbs (1362 kg) submerged and a dry weight of approximately $3437 \mathrm{lbs}$. The 3000-1b weight consists of a tase weight assembly and a top weight assembly, H-1-83994, Items 3 anr 4 , that fit together to forin the $3000 \mathrm{lb}$. test weight assembly. Two parts are required for the $3000 \mathrm{lb}$. test weight assembly to meet the $1700 \mathrm{lb}$. limit for equipment lowered into the $\mathrm{K}$ basins. Both parts of the $3000 \mathrm{lb}$. test weight assembly will be weighed separately in air (dry) and while submerged underwater.

The $570 \mathrm{~kg}$ test weight, H-1-83394, Item 2, is designed to provide an approximate $1256 \mathrm{lbs}$. (570 $\mathrm{kg}$ ) submerged and a dry weight of approximately $1440 \mathrm{lbs}$. The test weight will be certified in air (dry) and while submerged underwater.

Fabrication of the test weights is currently in process at the Site Fabrication Services fabrication shops at 200 West area with a completion date of 7/23/99. After fabrication the test weights will be delivered to a facility on the Hanford site where the certification of the test weights will be performed.

3.0 SCOPE

Energy NW will provide the following services:

3.1 Provide manpower necessary to perform the certifications. The buyer will provide a crane operator and a person to assist with the handling of the test weights. 
3.2 Certify the test weights. The test weights will be certified for dry (in air) weight and for subrerged (underwater) weight. The test weights will be certified to $+1-0.5 \%$. The following items will be certified:

- H-1-83994, Item 2, $570 \mathrm{~kg}$. Test Weight Assembly

- H-1-83994, Item 3, Base Weight Assembly

- H-1-83994, Item 4, Top Weight Assembly

3.3 Provide certification certificates for the two test weights items listed in 3.2. The certification certificates shall meet the requirements of HNF-PRO-490, Rev 1, Control of Measuring and Test Equipment.

3.4 The Hanford Standards Lab (HSL) assigned ID number shall be marked on the top of each test weight using $1 / 2 "$ high metal stamps.

3.5 The following outline represents the major steps for test weight certification. A certification test plan will be written to document the certification procecture.

3.5.1 The two test woights will be staged at the Hanford site facility selected by the buyer where the certification work will be performed.

3.5.2 Buyer will provide a calibrated dynamometer that will be used for all of the certification tests. The dynamometer will be calibrated to NIST traceable standards, HSL ID\# 545-29-06046, 0-2000 lb. range, and be accurate to +\% $0.1 \%$.

3.5.3 Buyer will supply the certified rigging devices (slings, etc) and water tank for perforsing this work.

3.5.4 Energy NW will install the dynamometer on the overhead crane and prepare it for use.

3.5.5 ise test weights will be lifted using the test weight spreader beam, $H-1-83 y j 4$, Item 7. The buyer will provide the spreader beam.

3.5.6 The spreader beam will be attached to the dynamometer and the dynamometer will be tared so that it indicates zero load.

3.5.7 The test weights will be lifted using the spreader beam and the dry weight will be measured and recorded. This will be repeated three times.

3.5.8 Repeat steps 3.4.5 and 3.4.6 for the remaining two weights.

3.5.9 Remove the dynamometer and spreader bearn. Reconnect the spreader beam to the overhead crane. Use the spreader beam to place the two test wejghts on the bottom of the water tank.

3.5.10 Disconnect the spreader bar from the crane.

3.5.11 The spreader bar will be connected to a sling (>2000 lb capacity), the other end of the sling will be connected to the bottom of the dynamometer and the crane hook to the top of the dynamometer. The sling must be of sufficient length to allow the dynamometer to be read when standing outside the water tank.

$$
\text { Pagez of } 4 \text { me t.1.17 }
$$


3.5.12 Lif the test weight that is being weighed so that it is free and clear of the tank bottom and any other obstacies. The tost weights and spreader bar must be completely submerged underwater. Determine a method that will allow you return to the same approximate hook elevation in the following steps.

3.5.13 Lower the test weight and disconnect the stiff back from the weight. Raise the stifiback to the same approximate elevation as in step 3.4.10.

3.5.14 Tare the dynamometer.

3.5.15 Lower the stiffback. Reconnect to the test weight and raise the weight to the same approximate hook elevation as in step 3.4.10.

3.5.16 Record the indicated submerged weight of the test weight.

3.5.17 Lower and raise the test weight two additional times and record the indicated weight.

3.5.18 Repeat steps 3.4.10 to 3.4.15 for the other two test weights.

3.5.19 Remove all two test weights from the water tank.

3.6 Re-calibrate the dynamometer used for the certification of the test weights.

\section{0 DELIVERABLES}

4.1 Certification certificate for each test weight. Certification certificates will indicate dry and submerged weight of each test weight.

4.2 Copy of calibration certificate for re-certification of the dynamometer used for the certification of the test weights.

\subsection{SCHRDULE}

The period of performance for this work is July 26,1999 to September 30, 1999. All certification/calibration work is targeted for completion by August 15, 1999, but shall be completed no later than September 30, 1999.

6.0 ADMINISTRATIVE

The safety class per HNF-PRO-704, "Hazard and Accident Analysis Process," is General Services and quality assurance level 3 is per HNF-PRO-259, Rev. 0., "Graded Quality Assurance."

A security badge, Level 0 , will be required by personnel performing this work.

7.0 OUAWTY ASSURANCE

Applicable quality assurance requirements and implementing procedures are identified in the "Spent Nuclear Fuel (SNF) Project Quality Assurance Program Plan" (QAPP).

\subsection{LNTERFACEPOINTS}

Energy NW shall report to the Project Engineer:

\subsection{DESH NTERFACE POINTS}


H.L. Roach/FDNW

FRS Deputy Manager (BTR)

FRS Sub-project Manager (Backup BTR)

FRS Design Authority
S. D. Godfrey ， 372-2927

J. M. Henderson 376-8926

B. D. Groth

\subsection{FDNW DNTERFACE POINTS}

FRS Engineering Manager Project Engineer

$\begin{array}{ll}\text { L. D. Kessie } & 376-1918 \\ \text { H.L. Roach } & 376-5595\end{array}$ 


\title{
ORIGINAL
}

\section{ENEROY

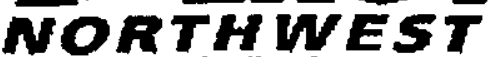 \\ Standards Laboratory}

Plant Support Facility

MD 1025, PO Bax 968

Richland, WA $99353-0968$

Phone (509) 377-8131 FAX (509) 377-8219

\section{Certificate of Calibration}

\author{
Manufacturer: SHOPMADE \\ Description: STAINLESS STEEL WEIGHT \\ Asset Number: 999-86-02-104 Serial Number: H-1-83994-030 \\ Report Number: 935663880 \\ Ref. Number: 75 \\ Customer: STECKER SO
}

\section{CALIBRATION INFORMATION}

\section{Test Conditions:}

Calibration Date: 26-Aug-99

Calibration Due: 26-Aug-00

Procedure / Rev: SLI 30-12 REV. 0

Technician: W. E. CALLAWAY

Remarks: $\quad$ CALIBRATED IN 305BLDG-USED AT K-BASIN

Test Results:

$\begin{array}{ll}\text { Pass: } & \mathbf{Y} \\ \text { Incomplete: } & \mathbf{N} \\ \text { Limited: } & \text { N } \\ \text { As Found: } & \text { Pass } \\ \text { As Left: } & \text { Pass }\end{array}$

\section{STANDARDS USED FOR CALIBRATION}

\begin{tabular}{llllll} 
Asset Number & Manufacturer & Model & & Description & Calibration Date \\
$545-29-06-046$ \\
\hline
\end{tabular}

\section{Notes/General Conditions:}

The standards and calibration program of the Energy Northwest Standards Laboratory complies with the requirements of 10 CFR50 Appendix B and ANSI/NCSL Z-540-1.

Unless otherwise noted:

The standards used in this calibration, described in the referenced calibration procedure with associated uncertainties or tolerances, are traceable to the Nationsl Institute of Standards and Technology (NIST). There are no special limitations of use imposed on this item.

This Report may not be reproduced, except in full, without the permission of the Energy Northwest Standards Laboratory.

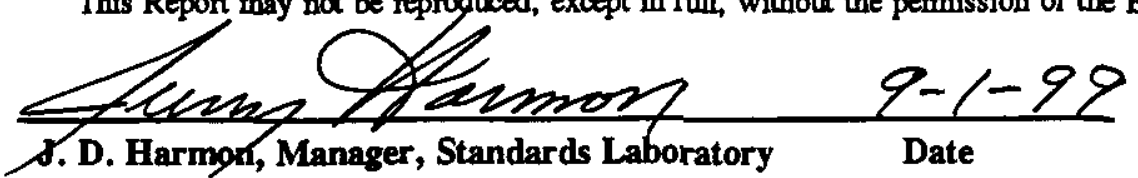




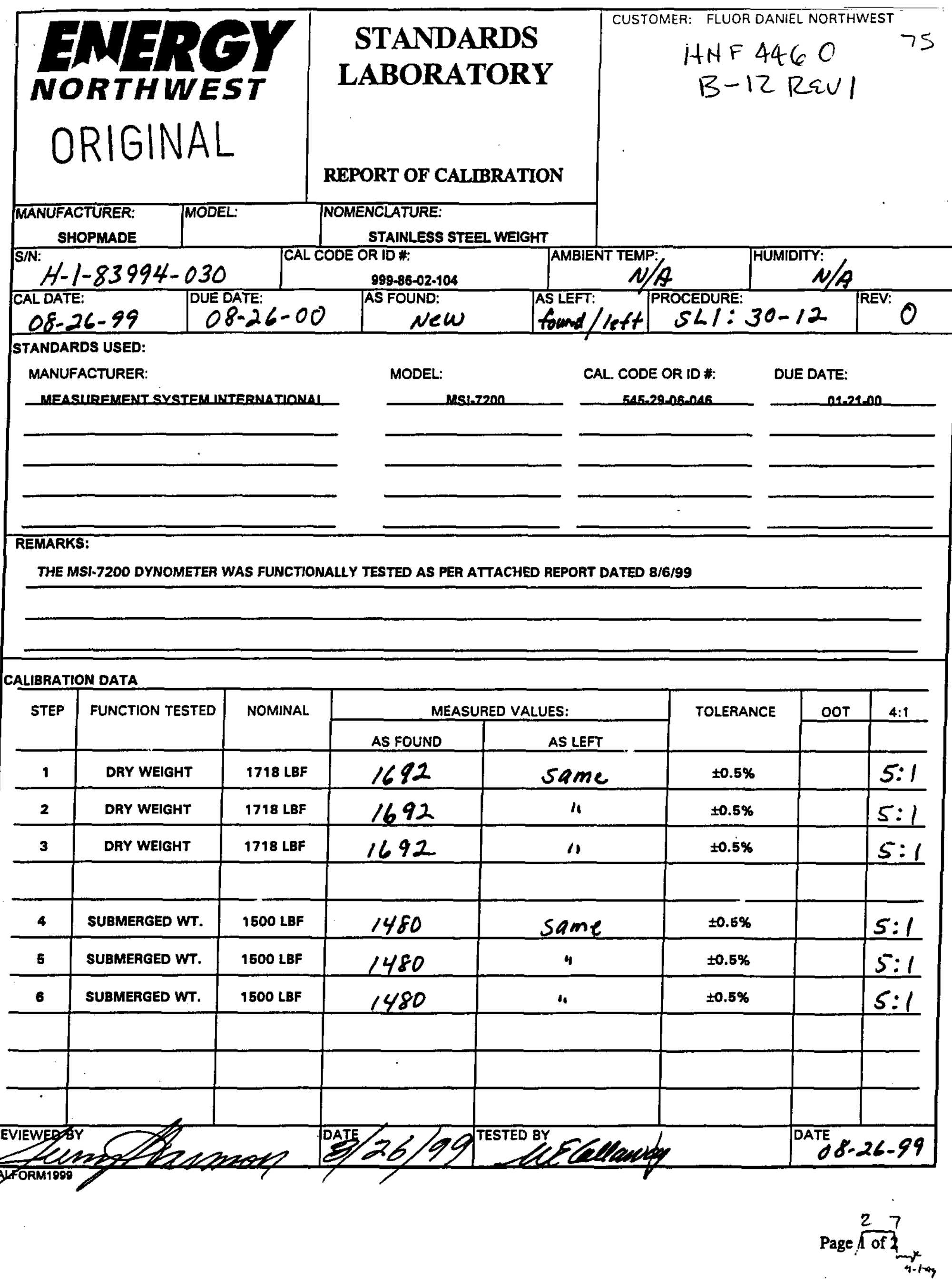




\section{STATEMENT OF WORK \\ FOR \\ Calibrtwoation of Test Weights for $\mathrm{K}$-Basin}

\subsection{OBJECTIVE}

This action is to provide for the certification of two test weights traceable to the National Institute of Standards Testing (NIST) standards. The test weights will be used at the K Basins to perform a daily calibration of the weighing system on the monorail mounted Multi-Canister Overpack (MCO) basket stiffback grapple.

All Hanford Site Measurement and Test Equipment (M\&TE) calibration/certification work is subcontracted by DynCorp to Energy NW. The certification of the test weight will be done by Energy NW.

\subsection{INTRODUCTION}

The Spent Nuclear Fuel (SNF) Project has been established to provide facilities and systems with which to retrieve, repackage, and interim store the spent nuclear fuel from the $K$ Basins in a safe configuration away from the Columbia River. The Fuel Retrieval System (FRS) Sub-project has been established to design, install, and test a system to repackage the spent nuclear fuel in the $K$ Basins. Fuel retrieval is accomplished using underwater process equipment. The SNF is loaded into $\mathrm{MCO}$ baskets and the MCO baskets are transferred into a $\mathrm{MCO}$ using the MCO basket stiffback grapple. The MCO basket stiffback grappje has an integral weighing system that is used to weigh the loaded MCO baskets. Test weights will be used to perform a daily accuracy and linearity check of the MCO basket stiffback grapple weighing system.

Two test weights have been designed to be used with the MCO basket stiffback grapple to provide the required daily linearity check of the weighing system.

The $3000 \mathrm{lb}$. test weight assembly, H-1-83994, ltem l, is designed to provide approximate 3000 lbs $(1362 \mathrm{~kg}$ ) submerged and a dry weight of approximately $3437 \mathrm{lbs}$. The $3000-1 \mathrm{~b}$ weight consists of a base weight assembly and a top weigill assembly, H-1-83994, Items 3 and 4, that fit togethes to form the $3000 \mathrm{lb}$. test weight assembly. Two parts are required for the $3000 \mathrm{lb}$. test weight assembly to meet the $1700 \mathrm{lb}$. limit for equipment lowered into the $\mathrm{K}$ basins. Both parts of the $3000 \mathrm{lb}$. test weight assembly will be weighed separately in air (dry) and while submerged underwater.

The $570 \mathrm{~kg}$ test weight, H-1-83394, Item 2, is designed to provide an approximate $1256 \mathrm{lbs}$. (570 $\mathrm{kg}$ ) submerged and a dry weight of approximately $1440 \mathrm{lbs}$. The test weight will be certified in air (dry) and while submerged underwater.

Fabrication of the test weights is currently in process at the Site Fabrication Services fabrication shops at 200 West area with a completion date of $7 / 23 / 99$. After fabrication the test weights will be delivered to a facility on the Hanford site where the certification of the test weights will be performed.

3.0 SCOPE

Energy NW will provide the following services:

3.1 Provide manpower necessary to perform the certifications. The buyer will provide a crane operator and a person to assist with the handling of the test weights. 
3.2 Certify the test weights. The test weights will be certified for dry (in air) weiglit and for submerged (underwater) weight. The test weights will be certified to $+1-0.5 \%$. The following items will be certified:

- H-1-83994, Item 2, $570 \mathrm{~kg}$. Test Weight Assembly

- H-1-83994, Item 3, Base Weight Assembly

- H-1-83994, Item 4, Top Weight Assembly

3.3 Provide certification certificates for the two test weights items listed in 3.2. The certification certificates shall meet the requirements of HNF.PRO-490, Rev 1, Control of Measuring and Test Equipment.

3.4 The Hanford Standards Lab (HSL) assigned ID aumber shall be marked on the top of each test weight using $1 / h^{\prime \prime}$ high metal stamps.

3.5 The following outline represents the major steps for test weight certification. A certification test plan will be written to document the certification procedure.

3.5.1 The two test weights will be staged at the Hanford site facility selected by the buyer where the certification work will be performed.

3.5.2 Buyer will provide a calibrated dynamometer that will be used for all of the certification tests. The dynamometer will be calibrated to NIST traceable staodards, HSL ID\# 545-29-06046, 0-2000 $\mathrm{lb}$. range, and be accurate to +1. $0.1 \%$.

3.5.3 Buyer will supply the certified rigging devices (slings, etc) and water tank for performing this work.

3.5.4 Energy NW will install the dynamometer on the overhead crane and prepare it for use.

3.5.5 The iest weights will be lifted using the test weight spreader beam, $\mathrm{H} \cdot 1-83994$, Item 7. The buyer will provide the spreader beam.

3.5.6 The spreader beam will be attached to the dynamometer and the dynamometer will be tared so that it indicates zero load.

3.5.7 The test weights will be lifted using the spreader beam and the dry weight will be measured and recorded. This will be repeated three times.

3.5.8 Repeat stops 3.4.5 and 3.4.6 for the remaining two weights.

3.5.9 Remove the dynamometer and spreader beam. Reconnect the spreader beam to the overhead crane. Use the spreader beam to place the two test weights on the bottom of the water tank.

3.5.10 Disconnect the spreader bar from the crane.

3.5.11 The spreader bar will be connected to a sling ( $>2000 \mathrm{lb}$ capacity), the other end of the sting will be connected to the bottom of the dynamometer and the crame hook to the top of the dynamometer. The sling must be of sufficient length to allow the dyramometer to be read when standing outside the water tank.

Page-20

me $\{\cdot 1 \cdot 5\}$ 
3.5.12 Lift the test weight that is being weighed so that it is free and clear of the tank bottom and any other obstacles. Tho test weights and spreader bar must be completely submerged underwater. Determine a method that will allow you return to the same approximate hook elevation in the following steps.

3.5.13 Lower the test weight and disconnect the stiff back from the weight. Raise the stiffback to the same approximate elevation as in step 3.4.10.

3.5.14 Tare the dynamometer.

3.5.15 Lower the stiffback. Reconnect to the test weight and raise the weight to the same approximate hook elevation as in step 3.4.10.

3.5.16 Record the indicated submerged weight of the test weight.

3.5.17 Lower and raise the test weight two additional times and record the indicated weight.

3.5.18 Repeat steps 3.4.10 to 3.4.15 for the other two test weights.

3.5.19 Remove all two test weights from the water tank.

3.6 Re-calibrate the dynamometer used for the certification of the test weights.

\section{0 DELIVERABLES}

4.1 Certification certificate for each test weight. Certification certificates will indicate dry and submerged weight of each test weight.

4.2 Copy of calibration certificate for re-certification of the dynamometer used for the certification of the test weights.

\section{S.0 SCHEDULE}

The period of performance for this work is July 26,1999 to September 30,1999 . All certification/calibration work is targeted for completion by August 15, 1999, but shall be completed no later than September 30, 1999.

\subsection{ADMINISTRATIVE}

The safety class per HNF-PRO-704, "Hazard and Accident Analysis Process," is General Services and quality assurance level 3 is per HNF-PRO-259, Rev. 0., "Graded Quality Assurance."

A security badge, Level 0 , will be required by personnel performing this work.

\subsection{OUALTY ASSURANCE}

Applicable quality assurance requirements and implementing procedures are identified in the "Spent Nuclear Fuel (SNF) Project Quality Assurance Program Plan" (QAPP).

\subsection{INTERFACE POINTS}

Energy NW shall report to the Project Engineer:

\subsection{DESH INTERFACE POINTS}


$999-8602-104$ HNF 4460 RaVI
$B-17$ 006

ORIGINAL

CSR-1. Rev 0

08/13/99

FRS Deputy Manager (BTR)

FRS Sub-project Manager (Backup BTR)

FRS Design Authority

S. D. Godfrey $\quad 372-2927$

J. M. Henderson $376-8926$

B. D. Groth $373-6673$

8.2 FDNW DNTERFACE PONTS

FRS Engineering Manager

Project Engineer

L. D. Kessie

376-1918

H.L. Roach

376-5595 


\section{ORIGINAL}

\section{ENEROY \\ NORTHWEST \\ Standards Laboratory}

Plant Support Facility

MD 1025, PO B0x 968

Richland, WA 99353-0968

Phone (509) 377-8131 FAX (509) 377-8219

\section{Certificate of Calibration}

$\begin{array}{rlrl}\text { Manufacturer: } & \text { SHOPMADE } & \text { Model: } & \text { NA } \\ \text { Description: } & \text { STADNLESS STEEL WEIGHT } & \\ \text { Asset Number: } & 999-86-02-105 & \text { Serial Number: } & \text { H-1-83994-020 } \\ \text { Report Number: } & 935664420 & \text { Ref. Number: } & 75 \\ \text { Customer: } & \text { STECKER SO } & & \end{array}$

CALIBRATION INFORMATION

\section{Test Conditions:}

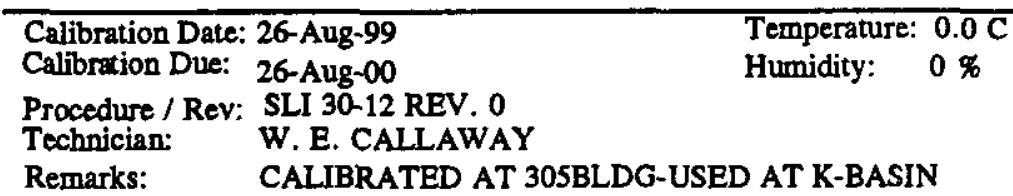

Test Results:

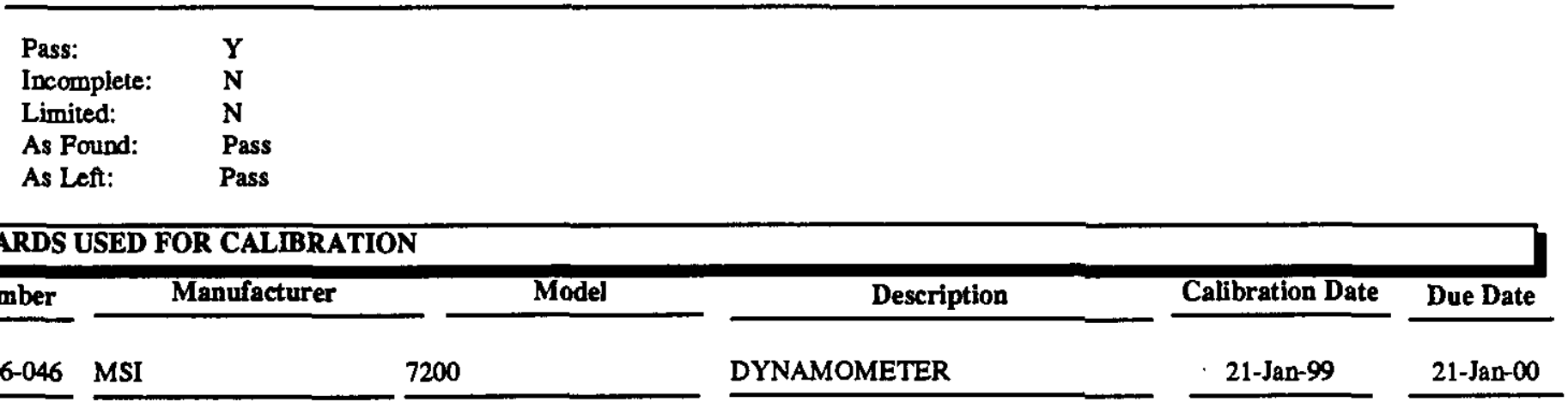

\section{Notes/General Conditions:}

The standards and calibration program of the Energy Northwest Standards Laboratory complies with the requirements of 10 CFR50 Appendix B and ANSI/NCSL Z-540-1.

Unless otherwise noted:

The standards used in this calibration, described in the referenced calibration procedure with associated uncertainties or tolerances, are traceable to the Nationgl Institute of Standards and Technology (NIST). There are no special limitations of use imposed on this item. This Report may not be reprodyced, except in full, without the permission of the Energy Northwest Standards Laboratory.

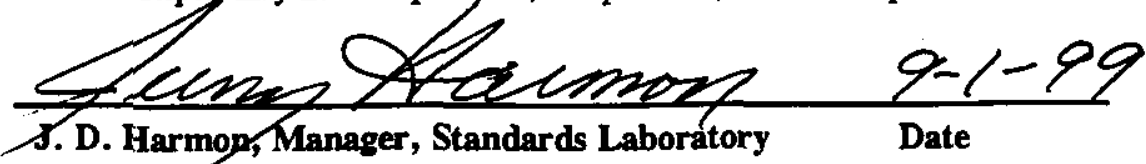


Fluor Daniel Hanford, inc.

P.O. Box 1000

Richland, WA 99352

Mal Involee To:

Eluor Dantel Bunford ATIN: ACCOUNYS PAYABLE G1-80 PO Box 1000 RICELAND WA 99352

Pleave Dixact Inquixier to:

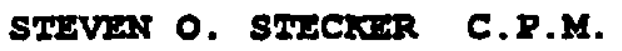

Title: CONHRACTING OEFICER

Phons: 509-373-7715

Ext:

Fax : 509-376-9016

Title: FRS - CAIIBRATION OF TRST WEIGETS EOR K-BASINS - ENERGY NA

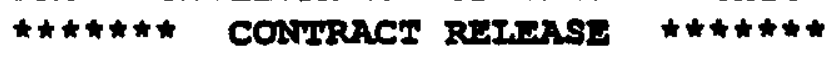

Totel value : $\$ 5,000.00$ USD

Pricing Wathod: Bstrupry

Contract Type : SaRvicas

Projact

Vendor Authortzed signature

Printed vam/Titlo

Date 8igned

\section{Vendor:}

Michaol I. Milson WASBIVTTON FUBIIC POWER SURPIY PO BOX $968 / \mathrm{MS}-055$ RICETAND WA 99352
Fork Iocution:

* NOT TO ExceEo **.

start Date: $07 / 26 / 99$

End Date : 09/30/99

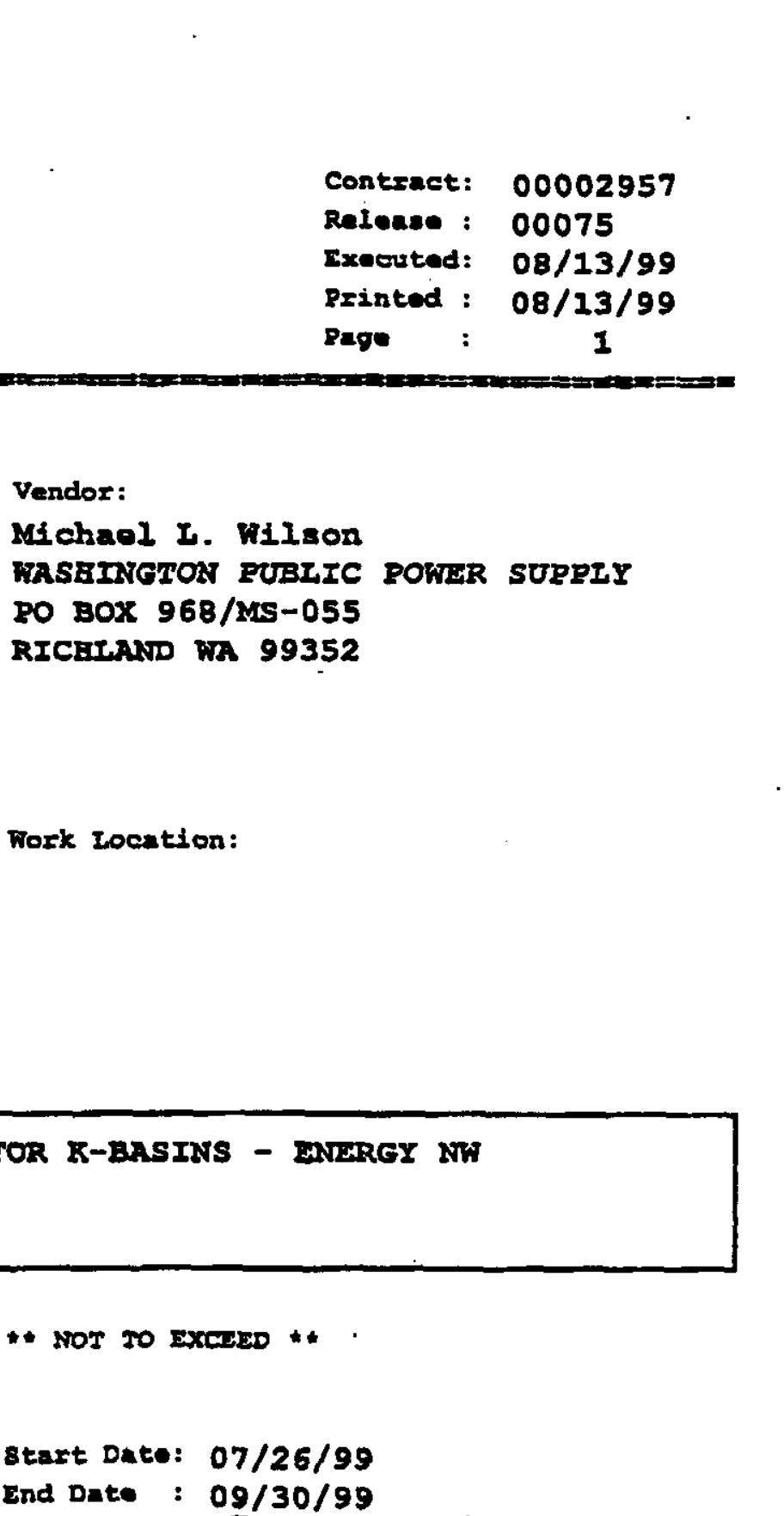




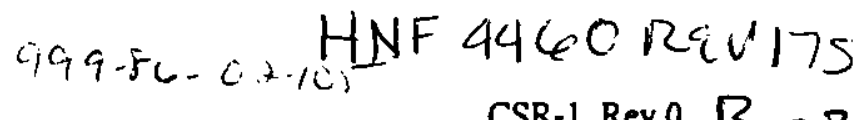

H.L. Roach/FDNW

\author{
STATEMENT OF WORK \\ FOR \\ Calibrtwoution of Test Weights for $\mathrm{K}$-Basin
}

\title{
1.0 OBNECTIVE
}

This action is to provide for the certification of two test weights traceable to the National Institute of Standards Testing (NIST) standards. The test weights will be used at the K Basins to perform a daily calibration of the weighing system on the monorail mounted Multi-Canister Overpack (MCO) basket stiffback grapple.

All Hanford Site Measurement and Test Equipment (M\&TE) calibration/certification work is subcontracted by DynCorp to Energy NW. The certification of the test weight will be done by Energy NW.

\subsection{INTRODUCTION}

The Spent Nuciear Fuel (SNF) Project has been established to provide facilities and systems with which to retrieve, repackage, and interim store the spent nuclear fuel from the K Basins in a safe configuration away from the Columbia River. The Fuel Retrieval System (FRS) Sub-project has been established to design, install, and test a system to repackage the spent nuclear fuel in the $\mathrm{K}$ Basins. Fuel retrieval is accomplished using underwater process equipment. The SNF is loaded into MCO baskets and the MCO baskets are transferred into a MCO using the MCO basket stiffback grapple. The MCO basket stiffback grapple has an integral weighing system that is used to weigh the loaded MCO baskets. Test weights will be used to perform a daily accuracy and linearity check of the MCO basket stiffback grapple weighing system.

Two test weights have been designed to be used with the MCO basket stiffback grapple to provide the required daily linearity check of the weighing system.

The $3000 \mathrm{lb}$. test weight assembly, H-1-83994, Item 1, is designed to provide approximate 3000 lbs (1362 kg) submerged and a dry weight of approximately $3437 \mathrm{lbs}$. The 3000-lb weight consists of a base weight assembly and a top weight assembly, H-1-83994, Items 3 and 4, that fit together to form the $3000 \mathrm{ib}$. test weight assembly. Two parts are required for the $3000 \mathrm{lb}$. test weight assembly to meet the $1700 \mathrm{lb}$. limit for equipment lowered into the $\mathrm{K}$ basins. Both parts of the $3000 \mathrm{lb}$. test weight assembly will be weighed separately in air (dry) and while submerged underwater.

The $570 \mathrm{~kg}$ test weight, H-1-83394, Item 2, is designed to provide an approximate 1256 lbs. (570 $\mathrm{kg}$ ) submerged and a dry weight of approximately $1440 \mathrm{lbs}$. The test weight will be certified in air (dry) and while submerged underwater.

Fabrication of the test weights is currently in process at the Site Fabrication Services fabrication shops at 200 West area with a completion date of $7 / 23 / 99$. After fabrication the test weights will be delivered to a facility on the Hanford site where the certification of the test weights will be performed.

3.0 SCOPE

Energy NW will provide the following services:

3.1 Provide manpower necessary to perform the certifications. The buyer will provide a crane operator and a person to assist with the handling of the test weights. 


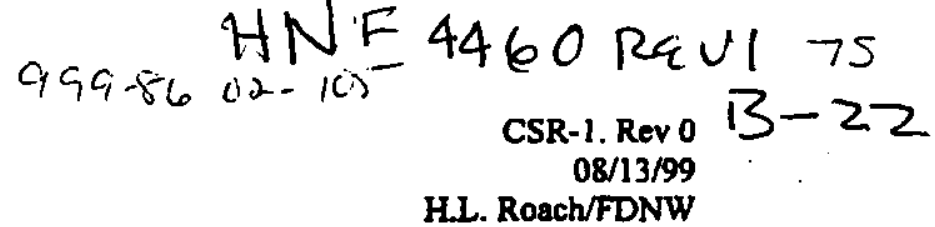

3.2 Certify the test weights. The test weights will be certified for dry (in air) weight and for submerged (underwater) weight. The test weights will be certified to $+1-0.5 \%$. The following items will be certified:

- H-1-83994, Item 2, $570 \mathrm{~kg}$. Test Weight Assembly

- H-1-83994, Item 3, Base Weight Assembly

- H-1-83994, Item 4, Top Weight Assembly

3.3 Provide centification certificates for the two test weights items listed in 3.2. The certification certificates shall meet the requirements of HNF-PRO-490, Rev 1, Control of Measuring and Test Equipment.

3.4 The Hanford Standards Lab (HSL) assigned ID number shall be marked on the top of each test weight using $1 / 4 "$ high metal stamps.

3.5 The following outline represents the major steps for test weight certification. A certification test plan will be written to document the certification procedure.

3.5.1 The two test woights will be staged at the Hanford site facility selected by the buyer where the certification work will be performed.

3.5.2 Buyer will provide a calibrated dymumometer that will be used for all of the certification tests. The dynamometer will be calibrated to NIST traceable standards, HSL ID\# 545-29-06046, 0-2000 lb. range, and be accurate to $+\%$ $0.1 \%$.

3.5.3 Buyer will supply the certified rigging devices (slings, etc) and weter tank for performing this work.

3.5.4 Energy NW will install the dynamometer on the overhead crane and prepare it for use.

3.5.5 The test weights will be liftod using the test weight syreader beam, H-1-83994, Item 7. Tho buyer will provide the spreader beam.

3.5.6 The spreader beam will be attached to the dynamometer and the dynamometer will be tared so that it indicates zero load.

3.5.7 The test weights will be lifted using the spreader beam and the dry weight will be measured and recorded. This will be repeated three times.

3.5.8 Repeat stops 3.4.5 and 3.4.6 for the remaining two weights.

3.5.9 Remove the dynamometer and spreader beam. Reconnect the spreador beam to the overhead crane. Use the spreader beam to place the two test wejghts on the bottom of the water tank.

3.5.10 Disconnect the spreader bar from the crane.

3.5.11 The spreader bar will be connected to a sling ( $>2000 \mathrm{lb}$ capacity), the other end of the sling will be connected to the bottom of the dynamometer and the crane hook to the top of the dynamometer. The sling must be of sufficient length to allow the dynamometer to be read when standing outside the water tank.

$$
\text { Page } 2 \text { of } 4-\pi+1.4
$$




$$
499.560 .105 \text { HNF } 4460_{R \varepsilon V I}
$$

3.5.12 Lift the test weight that is being weighed so that it is free and clear of the tank bottom and any other obstacles. Tho test weights and spreader bar must be completely submerged underwater. Determine a method that will allow you return to the same approximate hook elevation in the following steps.

3.5.13 Lower the test weight and disconnect the stiff back from the weight. Raise the stiffoack to the same approximate elevation as in step 3.4.10.

3.5.14 Tare the dynamometer.

3.5.15 Lower the stiffback. Reconnect to the test weight and raise the weight to the same approximate hook elevation as in step 3.4.10.

3.5.16 Record the indicated submerged weight of the test weight.

3.5.17 Lower and raise the test weight two additional times and record the indicated weight.

3.5.18 Repeat steps 3.4.10 to 3.4.15 for the other two test weights.

3.5.19 Remove all two test weights from the water tank.

3.6 Re-calibrate the dynamometer used for the certification of the test weights.

\section{0 DELIVERABLES}

4.1 Certification certificate for each test weight. Certification certificates will indicate dry and submerged weight of each test weight.

4.2 Copy of calibration certificate for re-certification of the dynamometer used for the certification of the test weights.

\subsection{SCHEDÜE}

The period of performance for this work is July 26, 1999 to September 30, 1999. All certification/calibration work is targeted for completion by August 15, 1999, but shall be completed no later than September 30, 1999.

\subsection{ADMINISTRATIVE}

The safety class per HNF-PRO-704, "Hazard and Accident Analysis Process," is General Services and quality assurance level 3 is per HINF-PRO-259, Rev. 0., "Graded Quality Assurance."

A security badge, Level 0 , will be required by personnel performing this work.

\subsection{OUALTY ASSURANCE}

Applicable quality assurance requirements and implementing procedures are identified in the "Spent Nuclear Fuel (SNF) Project Quality Assurance Program Plan" (QAPP).

\subsection{INTERFACE POINTS}

Energy NW shall report to the Project Engineer:

\subsection{DESH INTERFACE POINTS}


FRS Deputy Manager (BTR)

FRS Sub-project Manager (Backup BTR)

FRS Design Authority
S. D. Godfrey . 372-2927

J. M. Henderson $376-8926$

B. D. Groth 373-6673

L. D. Kessie

H.L. Roach
376-1918

376-5595
FRS Engineering

\subsection{FDNW DNTERFACE PONNTS}

\section{O8/13NW}

